ARTICLE

DOI: $10.1038 / s 41467-018-05367-2$

\title{
The actin cytoskeletal architecture of estrogen receptor positive breast cancer cells suppresses invasion
}

Marco Padilla-Rodriguez', Sara S. Parker (1) 1, Deanna G. Adams', Thomas Westerling², Julieann I. Puleo', Adam W. Watson', Samantha M. Hill', Muhammad Noon³, Raphael Gaudin (1) 4,5, Jesse Aaron (1) ${ }^{6}$, Daoqin Tong ${ }^{7}$, Denise J. Roe ${ }^{8}$, Beatrice Knudsen ${ }^{9} \&$ Ghassan Mouneimne ${ }^{1}$

Estrogen promotes growth of estrogen receptor-positive (ER+) breast tumors. However, epidemiological studies examining the prognostic characteristics of breast cancer in postmenopausal women receiving hormone replacement therapy reveal a significant decrease in tumor dissemination, suggesting that estrogen has potential protective effects against cancer cell invasion. Here, we show that estrogen suppresses invasion of ER+ breast cancer cells by increasing transcription of the Ena/VASP protein, EVL, which promotes the generation of suppressive cortical actin bundles that inhibit motility dynamics, and is crucial for the ERmediated suppression of invasion in vitro and in vivo. Interestingly, despite its benefits in suppressing tumor growth, anti-estrogenic endocrine therapy decreases EVL expression and increases local invasion in patients. Our results highlight the dichotomous effects of estrogen on tumor progression and suggest that, in contrast to its established role in promoting growth of ER+ tumors, estrogen has a significant role in suppressing invasion through actin cytoskeletal remodeling.

\footnotetext{
${ }^{1}$ Department of Cellular and Molecular Medicine, University of Arizona Cancer Center, University of Arizona, Tucson 85724 AZ, USA. ${ }^{2}$ Department of Medical Oncology, Division of Molecular and Cellular Oncology, and Center for Functional Cancer Epigenetics, Dana-Farber Cancer Institute and Harvard Medical School, Boston 02115 MA, USA. ${ }^{3}$ BioComputing Facility, University of Arizona, Tucson 85724 AZ, USA. ${ }^{4}$ INSERM U1110, Institut de Recherche sur les Maladies Virales et Hépatiques, Strasbourg 67000, France. ${ }^{5}$ Université de Strasbourg, Strasbourg 67000, France. ${ }^{6}$ Advanced Imaging Center, Howard Hughes Medical Institute, Janelia Research Campus, Ashburn 20147 VA, USA. ${ }^{7}$ School of Geography and Development, College of Social and Behavioral Sciences, University of Arizona, Tucson 85724 AZ, USA. ${ }^{8}$ University of Arizona Cancer Center and Mel and Enid Zuckerman College of Public Health, University of Arizona, Tucson 85724 AZ, USA. ${ }^{9}$ Department of Biomedical Sciences, Cedars-Sinai Medical Center, Los Angeles 90048 CA, USA.

Correspondence and requests for materials should be addressed to G.M. (email: gmouneimne@email.arizona.edu)
} 
E strogen receptor-positive $(\mathrm{ER}+)$ breast cancers are the most commonly diagnosed subgroup of breast tumors, and most breast cancer deaths are caused by metastatic ER+ tumors $^{1,2}$. Several lines of evidence suggest that the risk of ER+ breast cancer increases with estrogen exposure during a women's lifetime, for example, due to earlier menarche or late menopause (i.e., longer exposure to reproductive hormones due to longer ovarian activity) ${ }^{3}$. Moreover, large-scale clinical trials designed to look at the effects of hormone replacement therapy (HRT) on breast cancer incidence in postmenopausal women revealed that HRT increased the risk of breast cancer ${ }^{4,5}$. However, extended exposure to estrogen during HRT was associated with less dissemination and better outcome ${ }^{5}$. Interestingly, HRT did not reduce the locoregional recurrence rate $^{6}$, suggesting that under HRT, recurrent tumors are able to develop and grow locally at the initial tumor site but are less prone to disseminate and metastasize to distant sites.

In this study, we investigated this potential protective role of estrogen against cancer dissemination and metastasis. In a metaanalysis, including 17,497 patients from 10 clinical cross-sectional studies, we found that the metastatic burden in patients who developed breast cancer while on estrogen treatment was reduced. In addition, we found that ER is associated with lower invasive capacity. Despite the significant role of actin remodeling in cell invasion, the hormonal regulation of the actin cytoskeletal architecture in ER+ breast cancer cells, is not known. We found that ER promotes the formation of distinct actin structures with protective properties against invasion. We used a multimodal targeted discovery approach to examine the transcriptional regulation of actin cytoskeletal regulators by ER. Among a comprehensive list of known actin regulators, we identified a member of the Ena/VASP family of proteins, EVL, as the most significant transcriptional target of ER. We found that EVL is up-regulated in ER+ tumors and suppresses invasion, and that EVL levels are reduced in tumors after anti-estrogenic hormone therapy, which was associated with increased invasion.

\section{Results}

ER is associated with low breast cancer dissemination. In a meta-analysis of ten epidemiological studies ${ }^{6-15}$, we investigated lymph node (LN) positivity in postmenopausal women diagnosed with breast cancer while on HRT (current-users), compared to women who never used HRT (never-users). We used LN status as a metric of tumor cell dissemination and the commonly used cutoff of $>3(\mathrm{LN}>3)$ as a marker of distant metastasis beyond the regional axillary LNs. HRT was associated with lower odds ratio (OR) of having $\mathrm{LN}>3$, suggesting less distant dissemination (Fig. 1a, b). Moreover, to examine both regional and distant dissemination, we performed a binary assessment of LN status (LN+ or LN-). This analysis showed that OR of having LN+ was lower in HRT current-users, indicating less regional and distant dissemination (Supplementary Fig. 1a, b). In addition, in $\mathrm{ER}+$ breast cancer, tumors with low LN dissemination had higher ER levels, as compared to tumors with high LN dissemination (1.8-fold in $\mathrm{LN} \leq 3$ and 1.5-fold in $\mathrm{LN}-$, compared to $\mathrm{LN}>3$ and $\mathrm{LN}+$, respectively); not surprisingly, patients with less LNdisseminated tumors have higher survival rate (Supplementary Fig. 1c, d).

We investigated the effect of ER on cancer cell invasion, the initial step in metastatic dissemination, in breast cancer patient samples from two tissue microarrays (TMA\#1 and TMA\#2; see Methods). We determined the local invasion index (LII) for each tumor sample by employing the nearest neighbor distance (NND) approach, typically used in spatial analysis to study the secondorder effect or local variation of point patterns ${ }^{16}$. Treating cancer cells (identified by cytokeratin positivity) as stochastic events in a point pattern analysis, we measured the distance between the nucleus of each cell and the nucleus of its most proximal neighboring cell (NND) and calculated LII as the average NND within each tumor sample (Fig. 1c, d).

In TMA\#1, we analyzed 64 samples from ER+ and ERtumors, exhibiting a wide range of LN dissemination. Not surprisingly, compared to the more aggressive ER - tumors, ER+ tumors exhibited significantly lower LII (Fig. 1e, f), suggesting that ER positivity is associated with lower invasion rate. Moreover, tumors with LII $\leq 7 \mu \mathrm{m}$ (denoted as LII low) were associated with foldfold less LN dissemination than tumors with LII $\geq 9 \mu \mathrm{m}$ (denoted as LII high) (Supplementary Fig. 1e), suggesting that, in addition to ER positivity, low local invasion corresponds, as expected, to low distant LN dissemination. These data suggest that local invasion is a valid parameter to assess dissemination.

Furthermore, we investigated the association between ER expression and invasion in TMA\#2, which comprised samples from ER+ luminal B tumors. Among ER+ tumors, luminal B tumors disseminate more extensively and exhibit a wide range of ER expression levels ${ }^{17}$. Quantitative analysis of ER levels (average intensity of ER immunofluorescence in each tumor sample) and LII in 180 samples from TMA\#2 revealed a significant negative linear correlation between ER expression and invasion (Fig. 1g-i). In addition to expressing higher ER, low LII tumors are associated with less LN dissemination as compared to high LII tumors (Supplementary Fig. 1f). Moreover, analysis of survival rates in a cohort of patients with luminal B tumors ${ }^{18}$ shows that high ER is associated with better outcome (Supplementary Fig. 1g). Together, these data demonstrate that in luminal B breast cancer, tumors with high ER expression exhibit low dissemination and are less invasive.

To investigate the direct effect of altering ER activity on invasion, we developed an in vitro culture system that allowed us to quantify the level of invasion of ER+ MCF7 breast cancer cells into 3D matrix under estradiol (E2) or ER inhibitor treatment (Fig. 1j). We found that ER inhibition enhanced invasion of MCF7 cells, whereas E2 suppressed it (Fig. 1k, l). These results are consistent with previous reports of ER-suppressing invasiveness of breast cancer cells ${ }^{19,20}$. Conversely, as expected, ER inhibition suppressed proliferation of the cells, whereas E2 promoted proliferation (Supplementary Fig. 1h). Together, these results suggest that the observed changes in invasion upon altering ER activity are not the result of changes in the proliferation rate, but are rather induced by enhanced cell motility.

ER promotes suppressive cortical actin bundles. Using kymography analysis, we quantified the motility dynamics of membrane protrusion, which is crucial for cancer cell invasion ${ }^{21}$. This analysis showed a marked decrease in the number and speed of membrane ruffles in E2-treated MCF7 cells, as compared to control (Fig. 2a-c and Supplementary Movie 1). Conversely, protrusive activity was significantly enhanced at the leading edge of MCF7 cells treated with ER inhibitors (Fig. 2a-c). We validated these data in ER+ breast cancer T47D cells (Fig. 2d-f and Supplementary Movie 2). Mechanistically, enhanced motility dynamics are well-established characteristics of an aggressive invasive behavior ${ }^{22}$; therefore, the suppression of protrusive activity by ER is in line with the low dissemination of tumor cells with high ER expression.

Motility dynamics of membrane protrusions are regulated by the architecture of the actin cytoskeleton ${ }^{22}$. Examining actin cytoskeletal remodeling by ER, we found that E2-treated MCF7 cells exhibited prominent cortical actin bundles, which were 
concurrent with the absence of protrusions. Conversely, ER inhibition diminished these bundles and enhanced cell scattering and dissemination (Supplementary Fig. 2a, b and Supplementary Movie 3). Similar actin bundles have been previously observed in normal cells, where they constitute a contractile cortical barrier that suppresses Arp2/3-mediated protrusions ${ }^{23,24}$. We quantitated the levels of phosphorylated myosin light chain (pMLC), as a contractility marker ${ }^{25}$, and of Arp $2 / 3$ at the leading edge after altering ER activity in ER+ breast cancer cells. Considering the 3D morphometric properties of cortical bundles and protrusive ruffles, we used volumetric analysis of fluorescence intensity in confocal z-series (Fig. 2g). E2 treatment significantly a

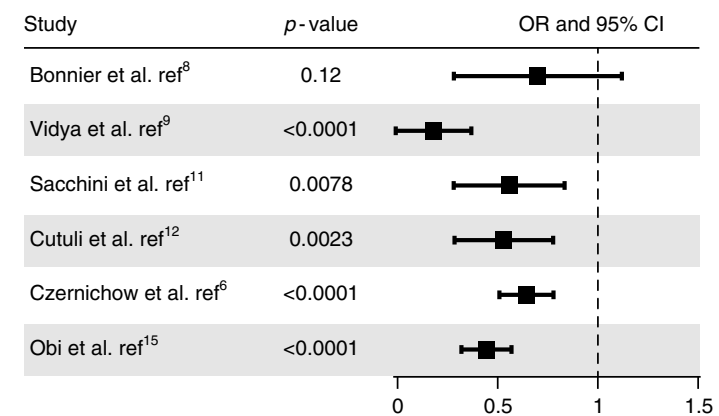

C

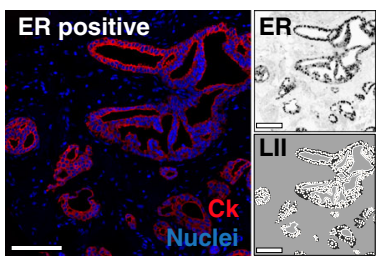

g
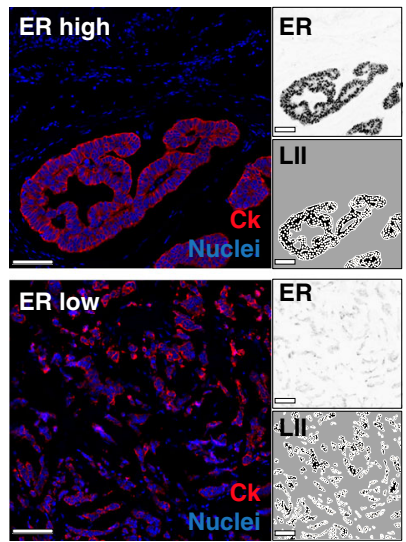

h b

\begin{tabular}{|c|c|c|c|}
\hline Study & & $\begin{array}{c}\text { Never- } \\
\text { users }\end{array}$ & $\begin{array}{c}\text { Current- } \\
\text { users }\end{array}$ \\
\hline \multirow{2}{*}{ Bonnier et al. ref ${ }^{8}$} & LN 0-3 & 210 & 116 \\
\hline & $\mathrm{LN}>3$ & 52 & 18 \\
\hline \multirow{2}{*}{ Vidya et al. ref ${ }^{9}$} & LN 0-3 & 173 & 55 \\
\hline & $\mathrm{LN}>3$ & 78 & 3 \\
\hline \multirow{2}{*}{ Sacchini et al. ref ${ }^{11}$} & LN 0-3 & 744 & 191 \\
\hline & $\mathrm{LN}>3$ & 150 & 20 \\
\hline \multirow{2}{*}{ Cutuli et al. ref ${ }^{12}$} & LN 0-3 & 336 & 239 \\
\hline & $\mathrm{LN}>3$ & 84 & 30 \\
\hline \multirow{2}{*}{ Czernichow et al. ref ${ }^{6}$} & LN 0-3 & 4598 & 1360 \\
\hline & $L N>3$ & 646 & 122 \\
\hline \multirow{2}{*}{ Obi et al. ref ${ }^{15}$} & LN 0-3 & 936 & 1383 \\
\hline & $\mathrm{LN}>3$ & 144 & 93 \\
\hline
\end{tabular}
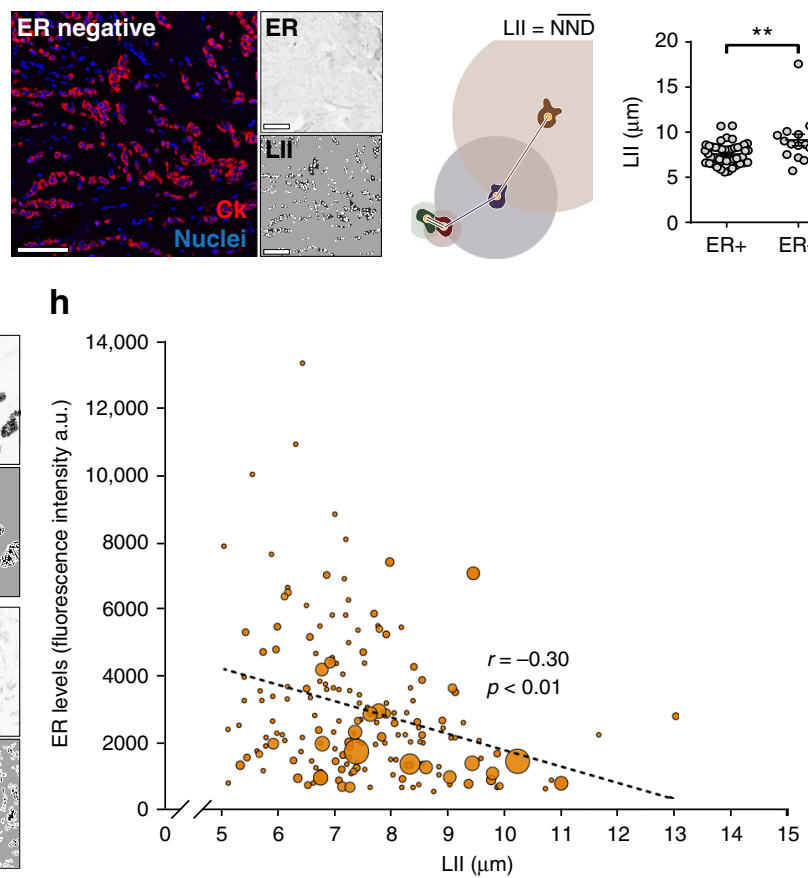

d

f

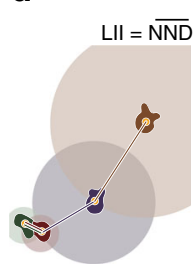

e

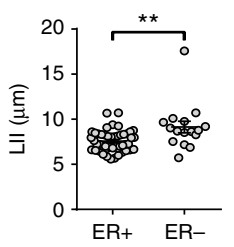

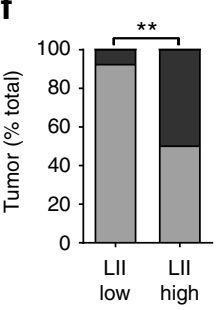

$\square \mathrm{ER}+$

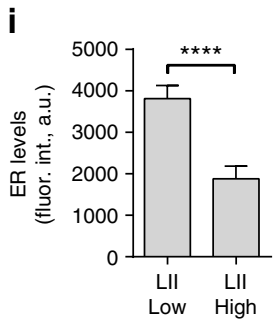

j

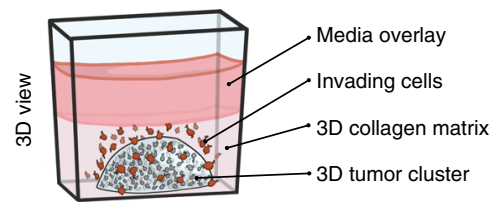

k

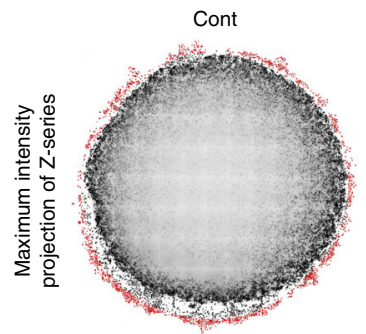

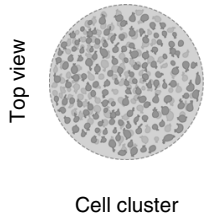

(before treatment)
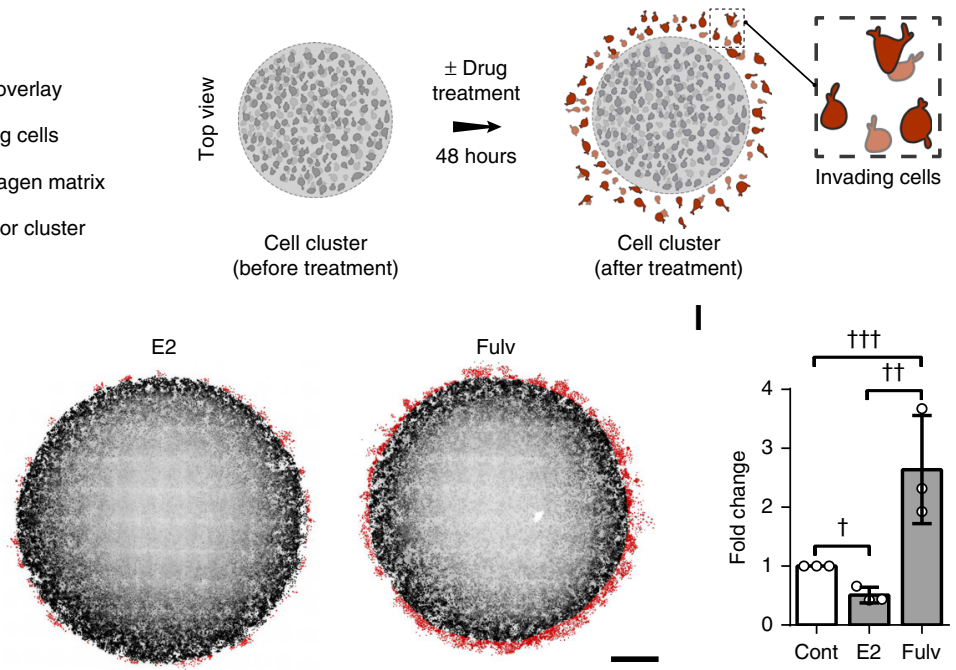

I

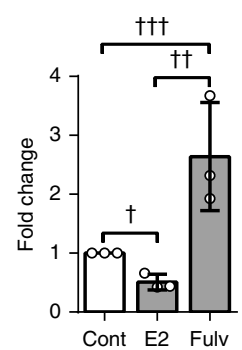


increased pMLC at cortical bundles, demonstrating elevated contractility, which was suppressed by ER inhibition (Fig. 2h, i and Supplementary Movie 4). The decrease in cortical contractility coincided with Arp2/3-positive leading edge ruffles; however, no significant changes in Arp2/3 levels at the leading edge were detected, suggesting that other actin regulators could be driving protrusion under ER inhibition (Fig. 2i). We confirmed these data in T47D cells (Fig. 2j, k). These results indicate that the suppressive effects of ER are mediated through remodeling of the actin cytoskeleton to generate suppressive cortical actin bunds (SCABs).

SCABs suppress leading edge motility. We further examined the relationship between cortical contractility and membrane motility dynamics. Using live-cell TIRFM, we imaged SCABs at the leading edge of MCF7 and T47D cells, before and after inhibiting contractility using the Rho-associated protein kinase (ROCK) inhibitor, Y-27632; the actin cytoskeleton was labeled with iRFPLifeact, and SCABs with MLC-mRuby2 (Supplementary Movie 5). Inhibiting contractility resulted in the loss of MLC at the leading edge and the dissolution of SCABs, and led to a rapid increase in protrusive activity, revealing a direct relationship between SCABs and the suppression of membrane motility dynamics (Fig. 3a, b). In addition, we validated the existence of SCABs, their contractile nature, and their suppressive effects in two types of epithelial cells: Madin-Darby canine kidney (MDCK) epithelial cells and Caco-2 human intestinal epithelial cells (Fig. 3c, d). Immunolabeling of pMLC in these cells revealed the contractile nature of these cortical structures, which corresponded with the absence of membrane protrusions (Fig. 3b). Importantly, the dissolution of SCABs dramatically increased the motility dynamics of the leading edge in both epithelial cell types (Fig. 3c). These data suggest that SCABs-and the attenuation of protrusive activity by SCABs-are present not only in ER+ breast cancer cells, but are common features of epithelial cells. ER is potentially enriching for SCABs by inducing the expression of actin regulators that promote SCAB formation.

$E V L$ is a transcriptional target of ER. Remodeling of the actin cytoskeleton is controlled by a multitude of actin regulators whose differential expression in cancer leads to distinct architectures that impact invasion ${ }^{26}$. To identify actin-binding proteins that are transcriptionally regulated by ER, we took a targeted discovery approach by examining the differential expression of a curated list of 285 actin regulators in ER+ vs. ER - tumors using 12 datasets $^{18,27-37}$ (Supplementary Data 1,2). The list of actin regulators was curated by first generating an extended list from the Gene Ontology (GO) Consortium using the broad search term "actin cytoskeleton," and then refining it by limiting the selection to genes that specifically express actin-binding proteins. In examining 12 independent microarray datasets, the Ena/VASP family member, EVL, was most differentially expressed in ER+ tumors, compared to ER- tumors (Fig. 4a, Supplementary Fig. 3a, and Supplementary Data 2, 3). This result was validated using the breast cancer The Cancer Genome Atlas (TCGA) RNAsequencing (RNA-seq) dataset (Supplementary Fig. 3b and Supplementary Data 4,5). Moreover, unsupervised clustering analysis of the RNA-seq data showed that EVL closely clustered with ESR1, the gene encoding ERa (Fig. 4b), and their expression exhibited the highest correlation index (Supplementary Data 6).

Strikingly, using ER chromatin immunoprecipitationsequencing (ChIP-seq) analysis, we found 12 high confidence ER peaks (defined in Methods) within the EVL gene and within $60 \mathrm{~kb}$ upstream of the transcription start site, suggesting a direct regulation of EVL by E2-stimulated ER (Fig. 4c). We further investigated four of the high confidence peaks by ChIPquantitative real-time PCR (qPCR) and two peaks showed significant enrichment in ER binding after E2 stimulation as compared to negative control targets (Fig. 4d and Supplementary Data 7). Importantly, EVL mRNA expression was induced by E2 in both MCF7 and T47D cells and was suppressed by ER inhibition (Fig. 4e and Supplementary Fig. 3c). In addition, using immunofluorescence labeling, we observed similar changes in EVL protein levels in MCF7 cells after altering ER activity with the different treatments (Fig. 4f, g). Together, these results demonstrate that $E V L$ is a bona fide transcriptional target of ER. Notably, EVL is a characterized suppressor of breast cancer cell invasion $^{26}$, making it an ideal candidate for mediating the protective effects of ER against invasion.

EVL promotes ER-mediated actin remodeling. EVL knockdown (KD), using validated EVL-targeting short hairpin (shRNA) ${ }^{26}$ (Supplementary Fig. 4a, b), rendered cells unresponsive to E2 by blocking the generation of SCABs and preventing the expected suppression of membrane motility dynamics by E2 treatment (Fig. 5a, b, Supplementary Fig. 4c-e, and Supplementary Movie 6). Conversely, overexpression of eGFP-EVL restored the generation of SCABs and suppressed protrusion under ER inhibition (Fig. 5c, d, Supplementary Fig. 5a-e, and Supplementary Movie 7). These results indicate that EVL is necessary and sufficient to generate ER-mediated SCABs and promote the suppressive effects of ER on membrane motility dynamics.

Both overexpressed eGFP-EVL and endogenous EVL (visualized in CRISPR-tagged eGFP-EVL cells and in EVL-

Fig. 1 ER expression is associated with low dissemination of breast cancer cells. a Meta-analysis of LN dissemination. Forest plot showing odds ratio, with $95 \%$ confidence interval $(O R, 95 \% \mathrm{Cl})$ of $L N>3$ in current-users compared with never-users of HRT. $\mathbf{b}$ Number of never-users and current-users with either LN $\leq 3$ or LN $>3$ in the studies analyzed in a. c Representative images of ER+ (left panel) and ER- (right panel) tumors from TMA\#1 (CDP-BCPTMA), labeled for cytokeratin (red) and nuclei (blue). Scale bar is $100 \mu \mathrm{m}$. Top-right inset shows ER labeling and bottom-right inset shows binary masks of cytokeratin stain (black) and nuclei (orange). d Illustration of local invasion index (LII) measurement. Connecting lines represent the nearest neighbor distance (NND) of illustrated cells (color of each line matches color of corresponding cell). LII is calculated as mean NND. e Quantification of LII in TMA\#1 (mean \pm s.e.m); ${ }^{\star \star} p=0.001$ (unpaired $t$ test). $\mathbf{f}$ Percentage of ER+ (gray) and ER- (black) tumors in low ( $\left.\leq 7 \mu \mathrm{m}\right)$ and high ( $\left.\geq 9 \mu \mathrm{m}\right)$ LII bins in TMA\#1; ${ }^{* *} p=0.002$ (unpaired $t$ test). $\mathbf{g}$ Representative images of luminal B breast tumors from TMA\#2 (Cedars-Sinai LumB TMA) with high (top panel) or low (bottom panel) ER expression. Top-right inset shows ER labeling and bottom-right inset shows binary masks of cytokeratin stain (black) and nuclei (orange). Scale bar is $100 \mu \mathrm{m}$. h Scatter plot of LII and ER levels in TMA\#2. For each data point, bubble area is proportional to the number of positive lymph nodes in the corresponding patient; $r$ is Pearson's correlation coefficient; correlation is significant at $p<0.01$. a.u. $=$ aribitrary units. $\mathbf{i}$ ER levels in tumors with low $(\leq 7 \mu \mathrm{m})$ and high $(\geq 9 \mu \mathrm{m})$ LII in TMA\#2; mean \pm s.e.m. ${ }^{\star \star \star \star} p<0.0001$ (Welch's $t$ test). $\mathbf{j}$ Illustration of 3D culture system for quantification of invasion in vitro. Cells embedded in central area invade into surrounding collagen matrix. Zoomed-in illustration of the boxed area shows invading cells in red. $\mathbf{k}$ Maximum intensity projections of confocal z-series of ER+ breast cancer MCF7 cells treated with drug vehicle, estradiol (E2), or fulvestrant (fulv). Binary mask (red) highlights invaded cells. Scale bar is $500 \mu \mathrm{m}$. I Quantification of invasion. Data are from three independent experiments; mean \pm s.d. $\dagger_{p}=0.003, \dagger p=0.01, \dagger \dagger p=0.03$ (unpaired $t$ test) 
immunolabeled control cells) localized at SCABs (Fig. $5 \mathrm{c}$ and Supplementary Fig. 6a, b). In addition, EVL was localized at SCABs in cell clusters embedded in $3 \mathrm{D}$ collagen matrix (Supplementary Fig. 6c). To further analyze EVL localization at SCABs, we imaged mEos2-EVL and actin using interferometric photoactivated localization microscopy (iPALM), which allows for super-resolution molecular localization (within $10 \mathrm{~nm}$ ) in the lateral and axial dimensions ${ }^{38}$. This analysis revealed that EVL enveloped the actin bundles in all dimensions at SCABs, as discerned in orthogonal projections of cross-sectional segments taken across different regions of the SCABs (Fig. 5e and Supplementary Fig. 6d). Moreover, EVL was highly enriched at the tips of SCABs, which extended into the focal adhesions; notably, at focal adhesions EVL localization was predominantly basal to actin (Fig. 5e and Supplementary Fig. 6d).

Interestingly, while inhibition of contractility dissolved SCABs and increased protrusive activity in control cells, EVLoverexpressing cells exhibited a higher threshold of sensitivity
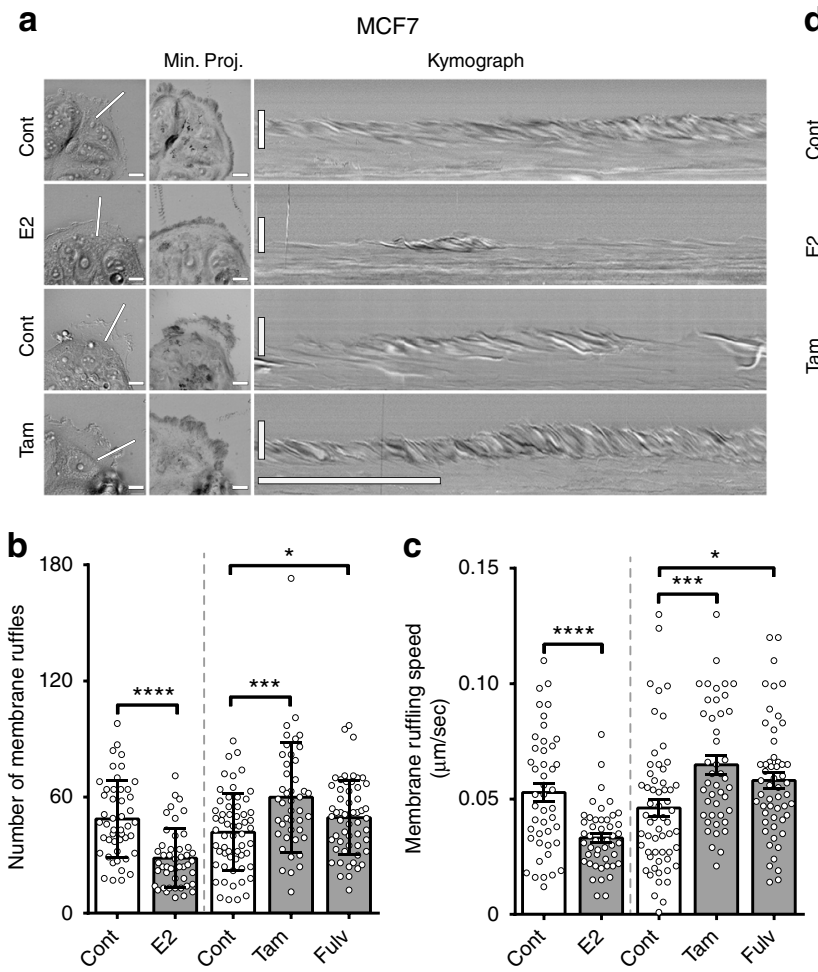

$\mathbf{h}$

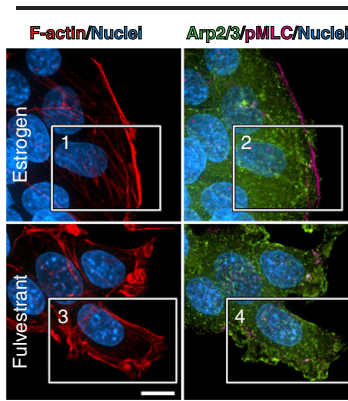

MCF7
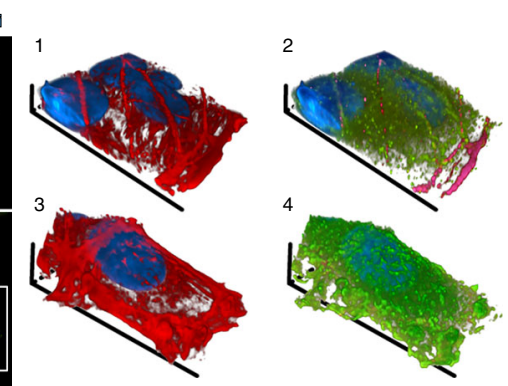

j

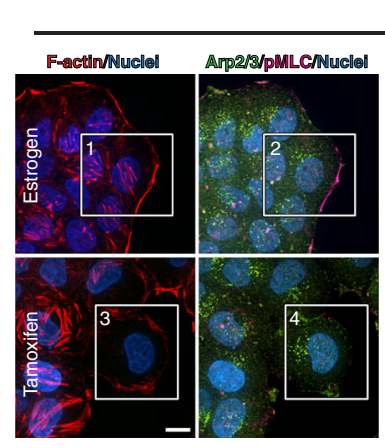

d

T47D
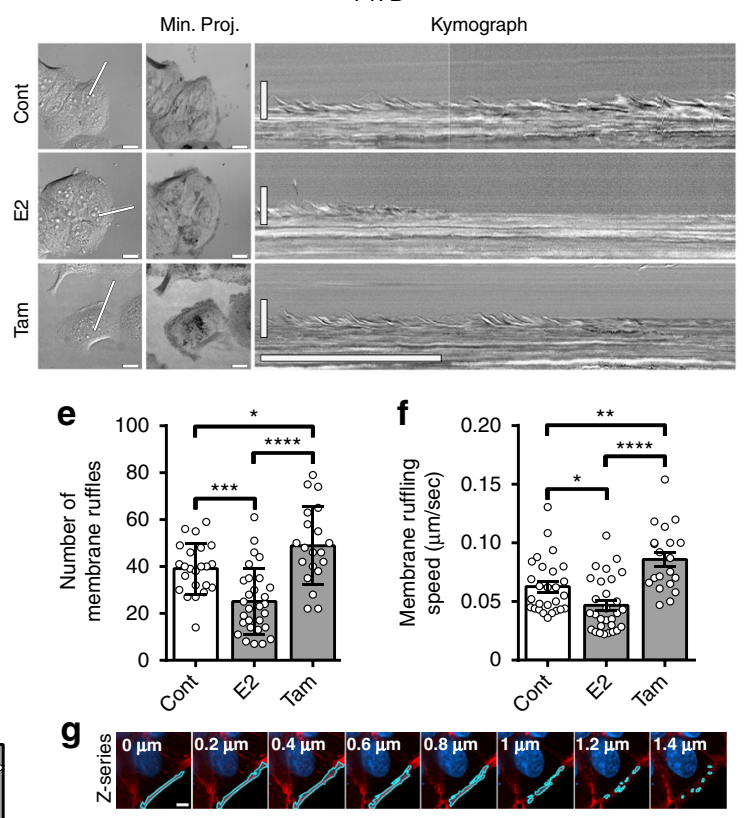

Confocal 3D reconstruction

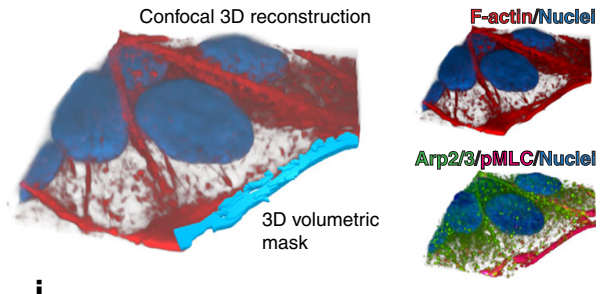

i$$
\text { Dे }
$$
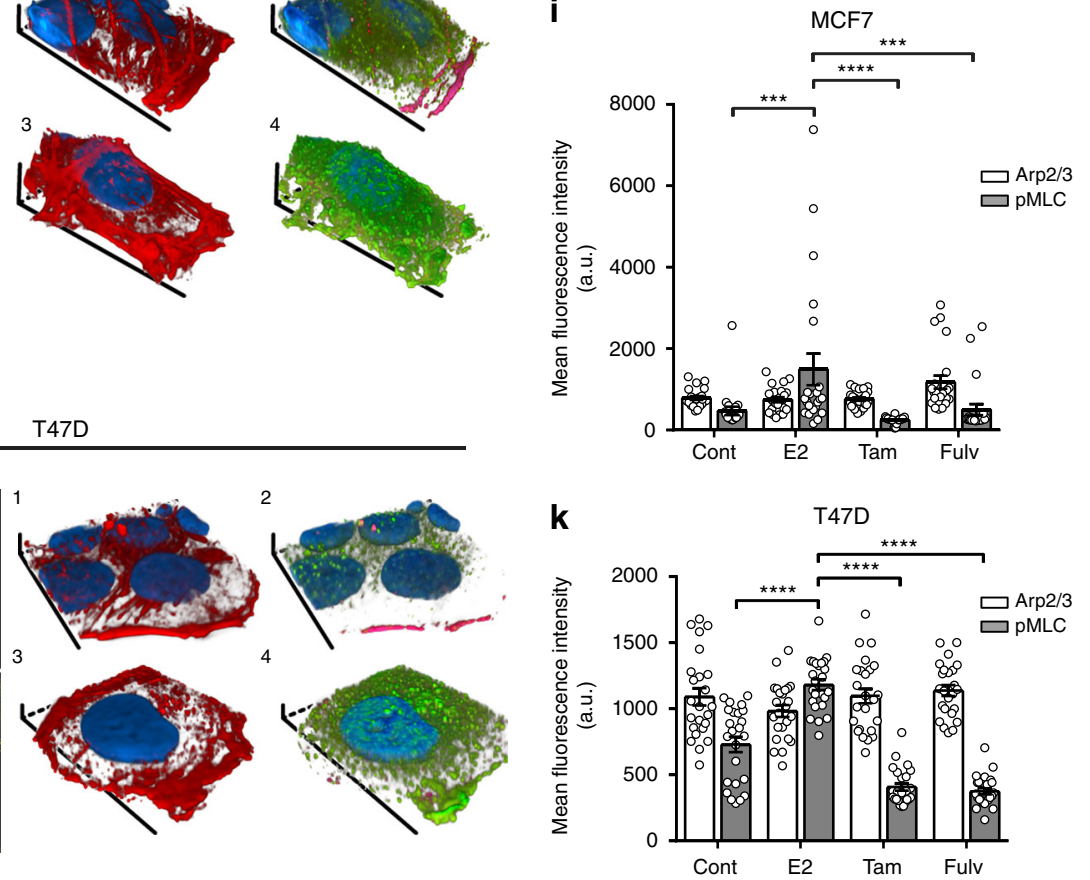
and their SCABs were more resistant to ROCK inhibition (Fig. 5f, Supplementary Fig. 5f, and Supplementary Movies 8, 9). This suggests that increased EVL expression not only leads to the enrichment of SCABs but also to the generation of more robust SCABs with higher efficacy in suppressing motility dynamics.

EVL promotes ER-mediated suppression of invasion. Importantly, EVL overexpression attenuated the increase in invasion induced by ER inhibition, whereas EVL KD reversed the suppressive effects of E2 on invasion (Fig. 6a, b). Moreover, EVL overexpression was sufficient to suppress invasion in ER- breast cancer SUM159 cells (Supplementary Fig. 7a, b). Together, these data suggest that EVL is necessary and sufficient to promote the E2-mediated suppression of invasion in vitro.

To extend these results in vivo, we examined the role of EVL in $\mathrm{ER}+$ breast cancer progression (BCP) using an intraductal MCF7 xenograft model $^{39}$. This model was used because it generates non-invasive ductal carcinoma in situ (DCIS) structures and maintains luminal differentiation, as opposed to fat-pad implantation, which induces mesenchymal differentiation ${ }^{39}$. Assessment of the intraductal tumors using a DCIS index (see Methods) showed that under E2 treatment, EVL KD constitutive (Fig. 6c and Supplementary Fig. 4a, b) and induced (Fig. 6d, e and Supplementary Fig. 7b) reduced the DCIS index and increased invasion compared to control. Consistent with the in vitro invasion data, these results show that EVL is necessary for mediating the suppression of invasion by ER in vivo.

EVL level is inversely correlated with invasion in ER+ tumors. Furthermore, analysis of immunofluorescence labeling of EVL and ER in luminal B tumor samples from TMA\#2 resulted in the following findings: first, EVL immunolabeling demonstrated the presence of SCABs in tumors (Supplementary Fig. 7d); second, expression analysis revealed that the levels of EVL and ER are positively correlated, to the extent that, within tumors exhibiting heterogeneity in ER expression, EVL expression changes with that of ER at the single-cell level (Fig. $6 \mathrm{f}-\mathrm{h}$ ); and third, tumors expressing low EVL exhibited high LII (Fig. 6i, j) and more distant dissemination (EVL was $\sim 30 \%$ less in tumors with $\mathrm{LN}>3$, $p=3 \times 10^{-5}$ ). Importantly, survival analysis showed that low EVL expression was associated with poor outcome in ER+ breast cancer in general, and in the luminal B subtype in particular (Fig. 6k and Supplementary Fig. 7e).
EVL level decreased after anti-estrogenic hormone therapy. We examined the effects of anti-estrogenic hormone therapy on EVL expression and invasion in ER+ breast cancer. We procured tumor samples from patients who were treated with neoadjuvant hormone therapy (Supplementary Data 8), and compared them to matching biopsy samples taken from the same tumors before therapy. We analyzed, at the single-cell level, invasion and EVL expression in a large population of tumor cells from three sets of before- and after-therapy tissue samples. Samples were collected from patients in stages 1,2 , and 3 , thus covering a range of invasive behaviors from different stages of progression. Importantly, the single-cell analysis approach allowed us to capture intratumoral heterogeneity and examine changes in population dynamics in response to treatment. EVL labeling revealed that prolonged anti-estrogenic therapy led to a dramatic decrease in EVL expression, which was associated with a decrease in ER (Fig. 7a, b and Supplementary Fig. 8a, b). Analysis of local invasion (LII) showed that tumors became more invasive after treatment (Fig. 7c, d). Particularly, the portion of highly invasive cells (LII high) increased after treatment, especially in stage 1 and stage 2 tumors, which exhibited a lower number of invasive cells prior to treatment (Fig. $7 \mathrm{c}, \mathrm{d})$. These data suggest that antiestrogenic hormone therapy, prescribed for these patients as neoadjuvant therapy to curb further growth of the tumors, potentially enhanced invasion with a marked decrease in EVL expression.

\section{Discussion}

Despite promoting growth of ER+ tumors, estrogen suppresses their invasion. We determined that this significant, but poorly recognized protective role of estrogen is mediated by a distinct ERdependent actin cytoskeletal architecture, reminiscent of actin organization in non-invasive epithelial cells ${ }^{23}$. As key features of these actin architectures, SCABs, which we denoted as SCABs, play a significant role in suppressing motility dynamics of the leading edge. Among a comprehensive list of actin cytoskeletal regulators, we found that $E V L$ is the most differentially expressed in ER+ tumors, is a direct transcriptional target of ER, and is essential for ER-mediated actin cytoskeletal remodeling and suppression of invasion in vitro and in vivo. This study establishes a novel mechanism by which ER restricts dissemination of cancer cells and, potentially, inhibits their metastatic capacity (Fig. 8).

ER enriches for SCABs in ER+ breast cancer cells by promoting EVL expression, potentially as part of the luminal tran-

Fig. 2 ER-mediated actin cytoskeletal remodeling induces suppressive cortical actin bundles (SCABs). a Leading edge kymography in representative timelapse movies (Supplementary Movie 1) of control and E2-treated MCF7 cells (cultured under hormone starvation conditions), and control and ER-inhibited cells (cultured in regular media). Left panels indicate position at which kymographs were registered (line), and middle panels show minimum intensity projections from entire time series (Min. Proj.); scale bar is $10 \mu \mathrm{m}$. Right panels show corresponding kymographs; vertical scale bar is $10 \mu \mathrm{m}$; horizontal scale bar is 5 min. b Membrane ruffle quantification; mean \pm s.d; ${ }^{\star} p=0.03,{ }^{\star \star \star} p=0.0005,{ }^{\star \star \star \star} p<0.0001$ (Welch's $t$ test). c Ruffling speed quantification; mean \pm s.e.m; ${ }^{\star} p=0.01,{ }^{\star \star \star} p=0.0007,{ }^{\star \star \star \star} p<0.0001$ (Welch's $t$ test). Data from three independent experiments ( $n \geq 45$ cells per group). $\mathbf{d}$ Leading edge kymography in representative time-lapse movies (Supplementary Movie 2) of T47D cells. e Membrane ruffle quantification; mean $\pm \mathrm{s} . \mathrm{d} ;{ }^{*} p=0.03$, ${ }^{\star \star \star} p=0.0002,{ }^{\star \star \star \star \star} p<0.0001$ (Welch's $t$ test). f Ruffling speed quantification; mean \pm s.e.m; ${ }^{\star} p=0.01,{ }^{\star \star} p=0.004,{ }^{\star \star \star \star} p<0.0001$ (Welch's $t$ test). Data from two independent experiments ( $n \geq 30$ cells per group). $\mathbf{g}$ Volumetric analysis of leading edge actin. Illustrative confocal $z$-series and corresponding 3D binary mask, demarcated as the area within $3 \mu \mathrm{m}$ of cell edge and used to quantify leading edge fluorescence intensity. $\mathbf{h}$ Maximum intensity projections of z-series of E2-treated or fulv-treated MCF7 cells (Supplementary Movie 4), and 3D reconstructions of boxed areas; F-actin (red), Arp2/3 (green), and pMLC (magenta). Scale bar is $10 \mu \mathrm{m}$. i Quantification of leading edge Arp2/3 and pMLC in MCF7 cells. Data from three independent experiments $(n=21$ cells per group; mean \pm s.e.m). By two-way ANOVA, interaction analysis shows significant differences at $p<0.0001$ ( $d f=3) ; \mathrm{pMLC}$ values are significantly different in E2-treated group compared to other groups $(p<0.001)$. Arp2/3 values are not significantly different between groups. $\mathbf{j}$ Maximum intensity projections of z-series of E2-treated or tam-treated T47D cells and 3D reconstructions of boxed areas. F-actin is red, Arp2/3 in green, and pMLC in magenta. Scale bar is $10 \mu \mathrm{m}$. $\mathbf{k}$ Quantification of leading edge Arp2/3 and pMLC in T47D cells. Data from three independent experiments $(n=21$ cells per group; mean \pm s.e.m). By two-way ANOVA, interaction analysis shows significant differences at $p<0.0001(\mathrm{df}=3)$; $\mathrm{pMLC}$ values are significantly different in E2-treated group compared to other groups $(p<0.001)$. Arp2/3 values are not significantly different between groups 

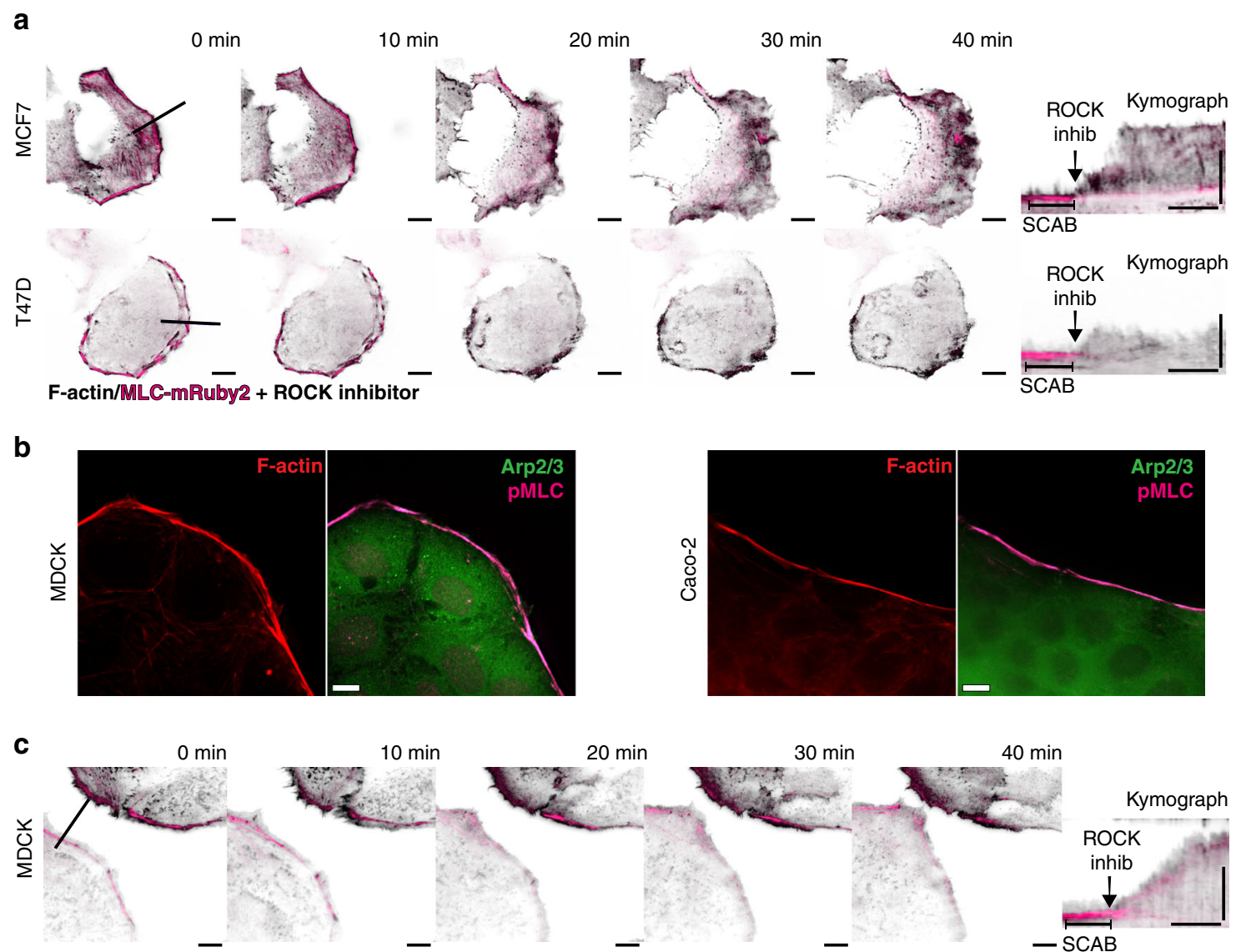

$30 \mathrm{~min}$

$40 \mathrm{~min}$

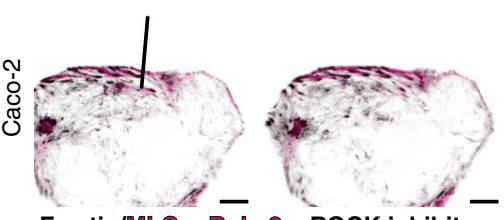

F-actin/MLC-mRuby2 + ROCK inhibitor

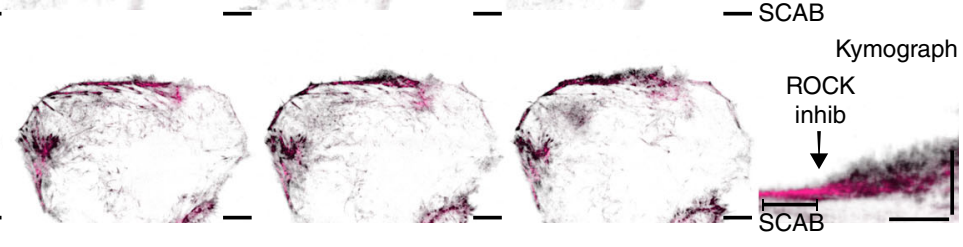

Fig. 3 SCABs suppress leading edge motility in a myosin contractility-dependent manner. a Leading edge kymography in MCF7 cells (top row) and T47D cells (bottom row) expressing iRFP-Lifeact (black) and MLC-mRuby2 (magenta) before and after dissolving SCABs using $25 \mu \mathrm{M}$ ROCK inhibitor. Left panels are images from TIRF microscopy time-lapse series before and after the addition of ROCK inhibitor (Supplementary Movie 5). Scale bar is 10 mm. Line shows the leading edge position at which kymographs were registered. Right panel shows MLC kymograph (denoting SCABs) superimposed over Lifeact kymograph (demarcating the leading edge). Vertical scale bar is $10 \mu \mathrm{m}$ and horizontal scale bar is 10 min. $\mathbf{b}$ Immunofluorescence of SCAB in MDCK epithelial cells and Caco-2 human intestinal epithelial cells. Representative images for F-actin, pMLC, and Arp2/3. Scale bar is 10 $\mu$ m. c Leading edge kymography in MDCK cells (top row) and Caco-2 (bottom row) cells expressing iRFP-Lifeact (black) and MLC-mRuby2 (magenta) before and after dissolving SCABs using $25 \mu \mathrm{M}$ ROCK inhibitor. Scale bar is $10 \mu \mathrm{m}$. Line shows the leading edge location at which the kymograph was registered. Right panel

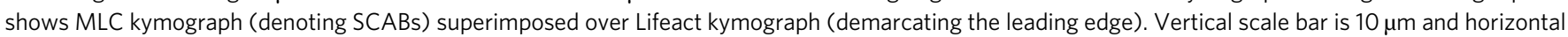
scale bar is $10 \mathrm{~min}$

scriptional program induced by ER in these cells. Indeed, EVL has been recently reported, by Tavares et al. ${ }^{40}$, to be high in luminal breast tumors. Another actin cytoskeletal regulator, VCL, has been described as a transcriptional target of $\mathrm{ER}^{20}$. However, VCL was not up-regulated in any of the clinical datasets that we have analyzed, and its expression was not correlated with ER in ER+ tumors (Supplementary Data 3-6). Therefore, VCL is not expected to have a global role in keeping invasion in check in ER + tumors; VCL could potentially be down-regulated in only a subset of these tumors resulting in a specific mode of invasion, such as amoeboid invasion.
Interestingly, Tavares et al. ${ }^{40}$ proposed that high levels of EVL are required for growth of pre-invasive tumors, but that $E V L$ needs to be down-regulated in order to transition to an invasive state. These results are in line with our model and suggest that dissemination of ER+ breast tumors is potentially suppressed by high levels of EVL during tumor growth. Therefore, in ER+ breast cancer, the two central components of metastasis, tumor growth and dissemination, are potentially regulated by antagonistic mechanisms, suggesting that the dissemination step is distinct from the growth phase; this concept is consistent with the significant recurrence rate $(30 \%)$ but long remission time 
( $>5$ years) of this breast cancer subtype ${ }^{41}$. Our study highlights an emerging paradigm that deviates from the simplistic view that metastasis is mediated by the deregulation of a primary signal transduction pathway globally associated with malignant transformation; rather, for metastasis to happen, cancer cells need reprogramming to acquire invasive properties and overcome antagonistic regulatory mechanisms promoting growth.

Importantly, most ER+ breast cancer patients receive extended adjuvant anti-estrogenic therapies. Despite the overwhelming evidence demonstrating the efficacy of these therapies in reducing a

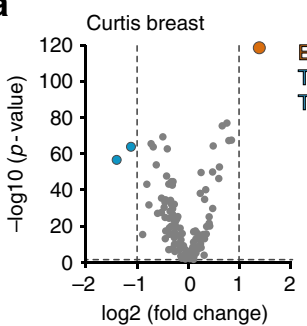

EVL TMSB15B TMSB15A

b

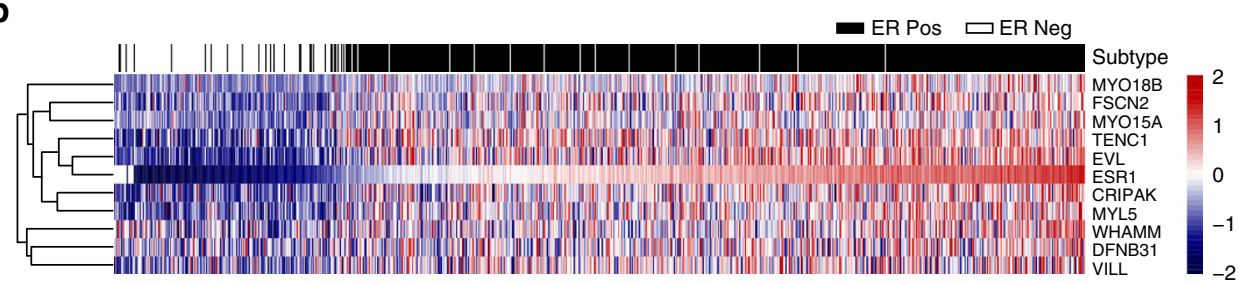

C
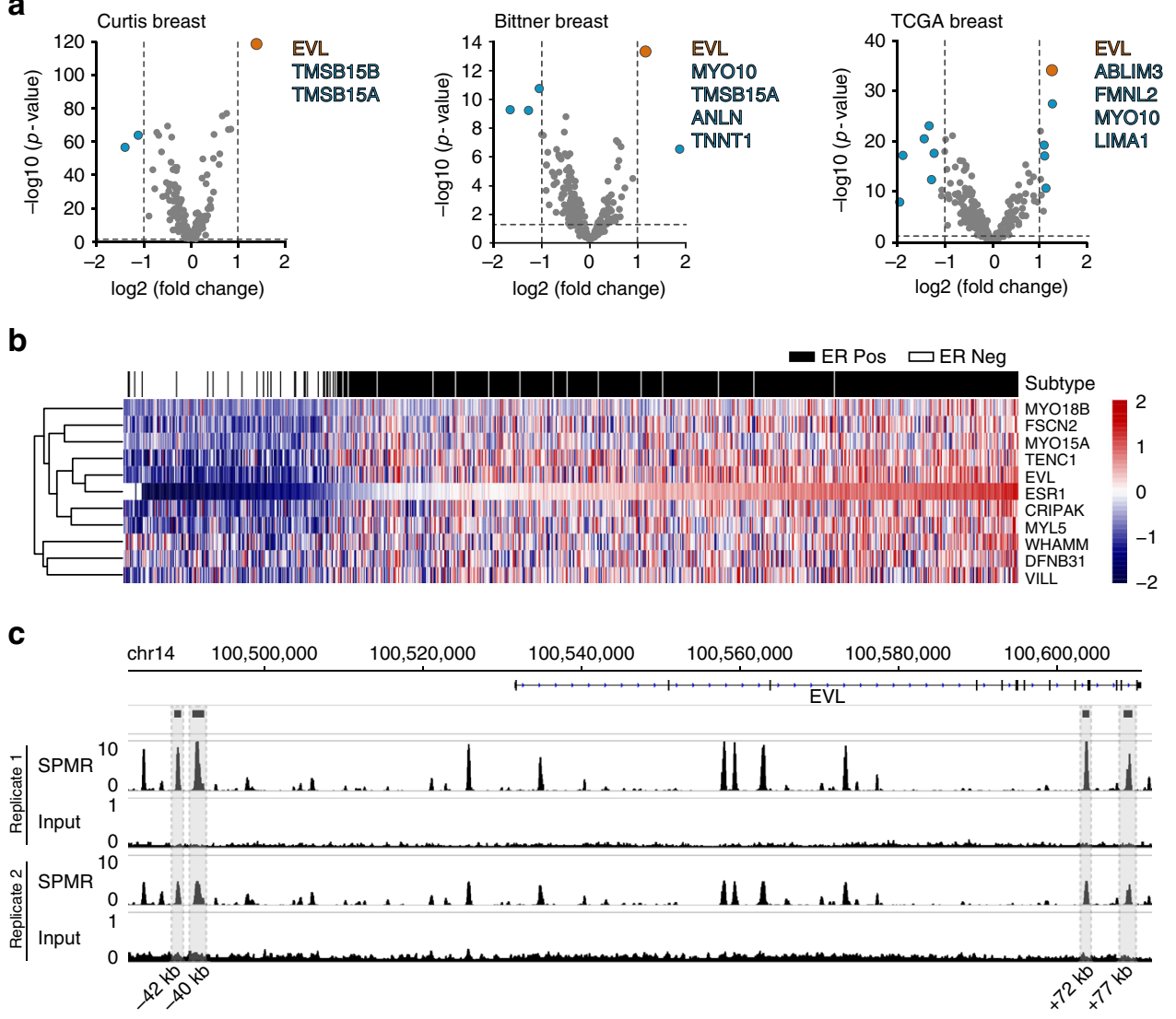

d

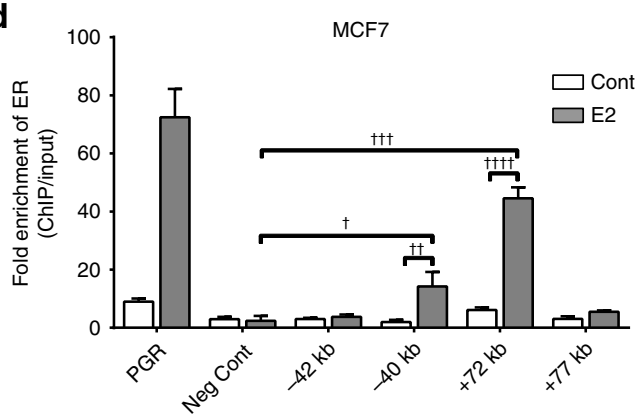

e

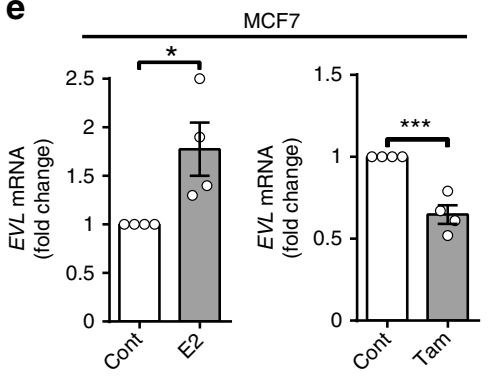

$\mathbf{f}$

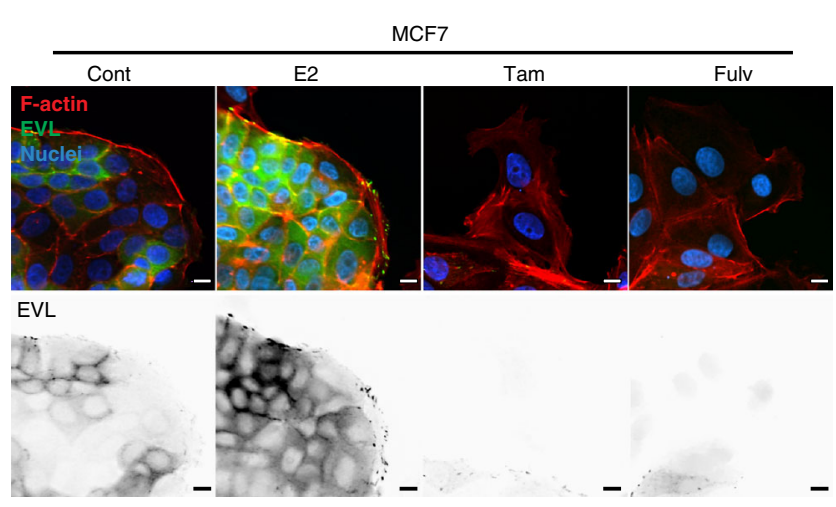

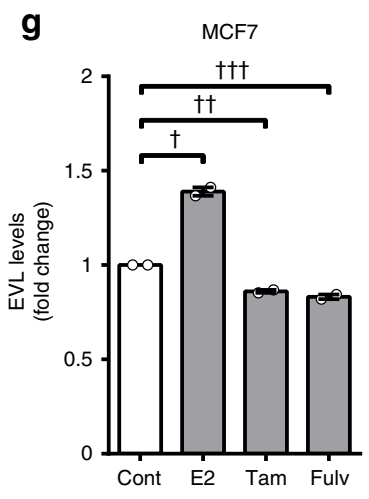


tumor recurrence, about one-third of treated patients are afflicted with recurrent, highly invasive tumors ${ }^{41}$. Our study raises the question of whether inhibiting ER could potentially downregulate EVL and aggravate tumor invasion and dissemination of these recurrent tumors. Finally, the clinical significance of this work is in advancing our understanding of the protective actions of estrogen against ER+ BCP.

\begin{abstract}
Methods
Antibodies and reagents. The following antibodies were used: human anti(Thr18/Ser19) pMLC 2 no. 3674 (Cell Signaling), hHuman anti-Arp2/3 clone no.13C9 (Millipore), human anti-pan cytokeratin no. AE1/AE3 (Dako), human anti-ER no. H4624 (Invitrogen), human anti-EVL no. HPA018849 (Sigma Prestige), human anti-EVL (a gift from Frank Gertler), and human anti-Actin (Abcam, ab3280). The following reagents were used: Alexa Fluor 568-Phalloidin (Thermo Fisher), Alexa Fluor 647-Phalloidin (Thermo Fisher), Hoechst 33342 (Thermo Scientific), $\beta$-estradiol (Sigma-Aldrich), tamoxifen (tam) (Sigma-Aldrich), fulvestrant (fulv) (Sigma-Aldrich), ROCK inhibitor ( $\gamma$-27632; Tocris), insulin growth factor 1 human recombinant (Sigma-Aldrich), puromycin (Thermo Scientific), G418 (Life Technologies), polybrene (EMD Millipore), and Fugene HD (Life Technologies)
\end{abstract}

Cell culture. MCF7 and HEK293T cells were cultured in high glucose Dulbecco's modified Eagle's medium (DMEM) base media with sodium pyruvate and L-glutamine (Corning) supplemented with additional $100 \mathrm{mM}$ L-glutamine, $10 \%$ fetal bovine serum (FBS; Gibco), and antibiotics (100 U/mL penicillin $+100 \mu \mathrm{g} / \mathrm{mL}$ streptomycin from Life Technologies, Inc.). T47D were cultured in RPMI1640 base media with L-glutamine (Corning) supplemented with additional $100 \mathrm{mM} \mathrm{L-glu-}$ tamine, $5 \mu \mathrm{g} / \mathrm{mL}$ insulin (Roche), 10\% FBS, and antibiotics. MCF10A were cultured in DMEM/F12 50/50 with L-glutamine (Corning), 5\% horse Serum, $20 \mathrm{ng} / \mathrm{mL}$ epidermal growth factor (Peprotech), $0.5 \mathrm{mg} / \mathrm{mL}$ hydrocortisone (Sigma), $100 \mathrm{ng} / \mathrm{mL}$ cholera toxin (Sigma), $10 \mu \mathrm{g} / \mathrm{mL}$ insulin, and antibiotics. Caco-2 were cultured in minimum essential media (MEM) base media with L-glutamine (Corning), 20\% FBS, and antibiotics. MDCK cells were cultured in high glucose DMEM base media with sodium pyruvate and L-glutamine (Corning), 10\% FBS, and antibiotics.

Plasmids. The following plasmids were used for RNAi: pLKO.1-TRC cloning vector was a gift from David Root (Addgene, plasmid \#10878), pLKO EVL shRNA (GE Dharmacon, TRCN0000063869, antisense TACTAGGATCTTCCATTTGGC), TRIPZ (GE Dharmacon, RHS4750), and TRIPZ EVL shRNA (GE Dharmacon, PN V3THS_300209, antisense TGGCTTTCATCTTCCTTCT), previously validated ${ }^{26}$. MSCV-eGFP-EVL was a gift from Frank Gertler, MIT, used for retroviral expression in kymography experiments. Lentiviral construct pLenti CMV-MRLC1mRuby2-IRES-PuroR was generated by PCR amplification of complementary DNA (cDNA) for Homo sapiens MRLC1 from eGFP-MRLC (a gift from Tom Egelhoff, Addgene, \#35680) and mRuby2-N1 (a gift from Michael Davidson, Addgene, \#54614) to create C-terminal-tagged MRLC. Fusion construct was subcloned into the expression vector pCIG3 (pCMV-IRES-GFP, a gift from Felicia Goodrum, Addgene, plasmid \#78264), modified to replace the GFP cassette with puromycin resistance gene. pCMV-iRFP670-EVL-IRES-BlastR was generated by PCR amplification of cDNA for Mus musculus EVL cDNA from MSCV-eGFP-EVL and piRFP670-N1 (a gift from Vladislav Verkhusha, Addgene, \#45457) to create $\mathrm{N}$-terminally tagged EVL. Fusion construct was subcloned into pCIG3 modified to replace GFP with blasticidin resistance. Lentiviral expression constructs pLenti EF1a-eGFP-EVL, pLenti EF1a-eGFP, pLenti Lifeact-iRFP670-BlastR, and pLenti
Lifeact-eGFP-BlastR were generated by Gateway technology (Thermo). pLenti EFla-eGFP-EVL and pLenti EFla-eGFP were generated by subcloning $M$. musculus EVL cDNA from MSCV-eGFP-EVL into eGFP-C2 (Clontech). eGFP and eGFP- $E V L$ were subcloned into Gateway entry vector pMuLE ENTR MCS L5-L2 (a gift from Ian Frew, Addgene, plasmid \#62085) modified to contain the hEFla promoter, and recombined with pMuLE ENTR MCS L1-R5 (a gift from Ian Frew, Addgene, plasmid \#62084) and pLenti Dest PuroR R1-R2 using LR Clonase II (Thermo) to generate the final lentiviral expression vector. pLenti Lifeact-eGFPBlastR and pLenti Lifeact-iRFP670-BlastR were generated by subcloning LifeactmEGFP (a gift from Michael Davidson, Addgene, \#54610), or Lifeact-iRFP670 into pMuLE ENTR MCS L1-R5, and recombined with pMuLE ENTR MCS L5-L2 and pLenti Dest BlastR. The following plasmids from this paper have been deposited to Addgene: pLenti CMV-MRLC1-mRuby2-IRES-PuroR (\#103031), Lifeact-iRFP670 (\#103032), pLenti Lifeact-iRFP670-BlastR (\#84385), pLenti Lifeact-eGFP-BlastR (\#84383), pENTR CMVie-Lifeact-iRFP670 L1-R5 (\#84390), pENTR CMVieLifeact-EGFP L1-R5 (\#84391), pLenti Dest BlastR R1-R2 (\#84574), and pLenti Dest PuroR R1-R2 (\#84575).

Virus production. HEK293T cells were transfected at $60 \%$ confluence using Fugene HD (Promega) in OptiMEM (Corning) with transfer plasmid and secondgeneration lentiviral packaging system (psPAX2 and pMD2.G, Addgene \#12260 and \#12259, gifts from Didier Trono) or pCL-Ampho (Novus) for lentiviral or retroviral production, respectively. Virus was collected $48-72 \mathrm{~h}$ post transfection, clarified by $0.45-\mu \mathrm{m}$ filters. Recipient cells were infected at $50 \%$ confluence with virus at a 1:1 dilution with their culturing media containing polybrene $(10 \mu \mathrm{g} / \mathrm{mL})$. Puromycin selection was started $36 \mathrm{~h}$ post infection.

Analysis of LN dissemination. LN dissemination was determined in a metaanalysis of epidemiological studies ${ }^{6-15}$ that investigated the effects of HRT on the clinical and prognostic characteristics of tumors in cohorts of breast cancer patients. Specifically, only studies that reported LN status in HRT current-users and never-users, with a minimum of 10 patients per treatment group, were included. Studies that investigated the effect of HRT on breast cancer incidence in healthy women were not considered. This focused approach enriched for breast cancer patients on HRT, providing sufficient data for a robust quantification of LN dissemination under estrogen treatment. For patient inclusion criteria, the analysis included only postmenopausal breast cancer patients (older than 50 years of age and had at least 6 months of amenorrhea or had double oophorectomy), who were on HRT for at least 6 months before diagnosis (denoted as current-users) or who never used HRT (denoted as never-users); HRT included treatment with estrogen alone or estrogen plus progestin. Patients taking estriol, a partial ER agonist available as a dietary supplement, were excluded from our analysis. This metaanalysis consisted of two parts: calculating OR for having more than three positive LN (LN >3), using six studies $6,8,9,11,12,15$; and calculating OR for having any number of positive LN (LN+), using nine studies ${ }^{7-15}$. For both analyses, Fisher's exact test was conducted to determine significance of each study. The OR was determined for each study and the exact $95 \%$ confidence interval was calculated using SATA 14.0.

Tissue immunolabeling. Slides were baked for $1 \mathrm{~h}$ at $60^{\circ} \mathrm{C}$, deparaffinized in xylene, and rehydrated in increasingly diluted ethanol solutions. Heat-induced epitope retrieval was performed ( $40 \mathrm{~min}$ boiling $+20 \mathrm{~min}$ cooling) in Tris-EDTA $\mathrm{pH} 9$ buffer for anti-ER (Invitrogen, 1:50 dilution) and anti-EVL (Sigma Prestige, 1:50 dilution) antibodies and sodium citrate $\mathrm{pH} 6$ buffer for anti-pan cytokeratin AE1/AE3 (Dako, 1:100 dilution). Following standard immunofluorescence staining protocol, samples were mounted in ProLong Diamond Antifade (Thermo Fisher) and allowed to cure for at least $24 \mathrm{~h}$ before imaging.

Fig. $4 \mathrm{EVL}$ is a transcriptional target of ER. a Differential gene expression analysis of actin cytoskeletal regulators in ER+ vs. ER- breast tumors. Volcano plot of significance ( $-\log _{10}$ of $p$ value) vs. $\log _{2}$ fold change. Horizontal dashed line represents threshold for significance at $p<0.05$. Vertical dashed lines represent threshold for positive or negative twofold change in gene expression. Insets are lists of genes that passed both thresholds in descending order of significance (lists were limited to five genes; full data analyses are presented in Supplementary Data 3). b Unsupervised clustering analysis of TCGA RNAseq dataset. Heatmap, generated by row scaling, showing expression of genes in the ESR1 cluster. Tumor samples were classified into ER+ (black) and ER(white) based on ER status (paired $t$ test used to determine significance). c Analysis of ER binding by chromatin immunoprecipitation (ChIP). Genomic region surrounding EVLshowing ER-ChIP sequencing profile as SPMR (signal per million reads) trace of two independent ER-ChIP sequencing samples, generated by MACS2.0 and visualized by IGV (Integrative Genomics Viewer). Corresponding input profiles are shown at $\times 10$ scale to clearly show the input DNA openness bias. d Fold enrichment of ER binding at four peaks found by ChIP-seq, in addition to positive (PGR) and negative controls. Fold enrichment is calculated as [DNA]-normalized ChIP/Input of $2^{\Delta \Delta C t}$. Values are means of data from four replicates; mean \pm s.e.m.; $p$ values were generated by paired $t$ test comparing E2 treated to vehicle treated and E2 treated to negative control; ${ }^{\dagger} p=0.009,{ }^{\dagger} p=0.007, \omega^{\dagger} p=0.001, i t i p=0.001$. e Analysis of the Regulation of EVL expression by ER. qPCR of EVL mRNA in MCF7 cells treated with corresponding drugs for $24 \mathrm{~h}$, normalized to GAPDH. Fold change shown between treatment groups. Values are means of data from four independent experiments; mean \pm s.e.m.; ${ }^{\star} p=0.03$, ${ }^{\star \star \star} p=0.0008$ (unpaired $t$ test). $\mathbf{f}$ Immunolabeling of MCF7 cells after corresponding drug treatment for $72 \mathrm{~h}$. Bottom row shows EVL labeling. Scale bar is $10 \mu \mathrm{m}$. g Quantification of EVL levels in MCF7 cells after treatment with corresponding drugs for $72 \mathrm{~h}$. Values are means of data pooled from two independent experiments; mean \pm s.e.m. ${ }^{\dagger} p=0.03, \dagger^{\dagger} p=0.03,{ }^{\dagger \dagger} p=0.04$ (Welch's $t$ test) 
Imaging: Tumors were imaged using a $\times 20$ Plan Apo $0.75 \mathrm{NA}$ Nikon objective on a Nikon Ti-E inverted microscope equipped with a Hamamatsu ORCA-Flash $4.0 \mathrm{~V} 2$ cMOS camera, motorized stage, and maintained in focus using Nikon Perfect Focus System. Large composite images of whole tumor sections were generated by digital stitching using Nikon Elements. Tumor sections that were damaged or that exhibited high degree of inflammatory cell infiltration were excluded from the analysis.

LII analysis. Breast cancer TMAs: Two independent breast cancer TMA sets were used in this analysis: (TMA\#1) Cancer Diagnostics Program (CDP) BCP TMA from the National Cancer Institute (NCI), comprised of an array of ER + and ER - tumors and (TMA\#2) luminal B breast cancer TMA from Cedars-Sinai Medical Center (Dr. Johnathon Kaye), comprised of an array of luminal B breast cancer tumors with varied levels of ER expression. LN status was indicated in the corresponding pathology report for each patient. Samples that were damaged or that exhibited high degree of inflammatory cell infiltration were excluded from the analysis.

Matched before and after endocrine therapy patient samples: Samples were obtained from the Arizona Cancer Center Biospecimen Repository. Samples were collected in compliance with the University of Arizona Institutional Review Board policies. a

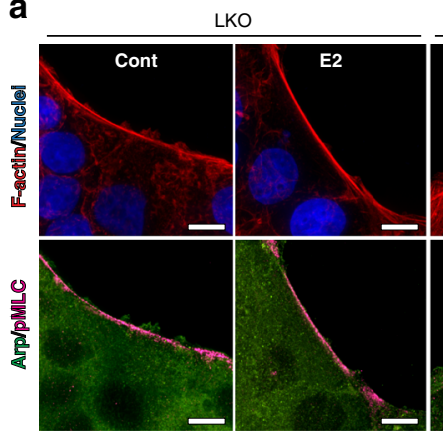

C

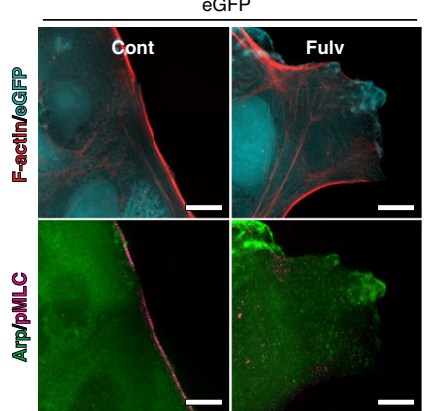

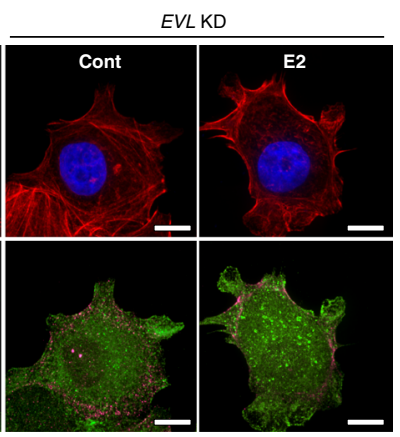

eGFP-EVL

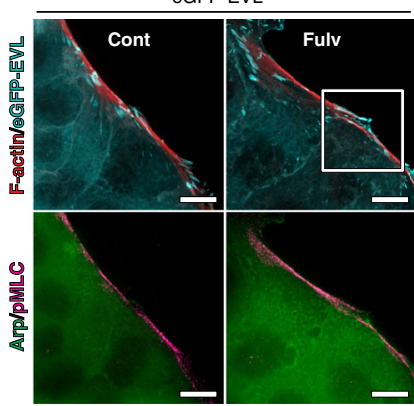

b

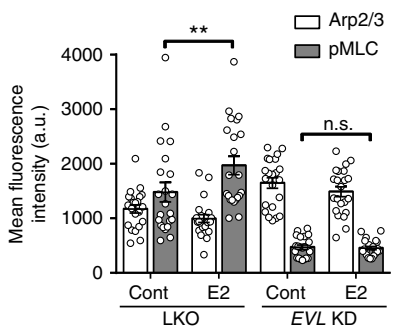

d

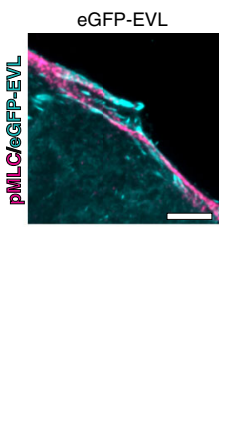

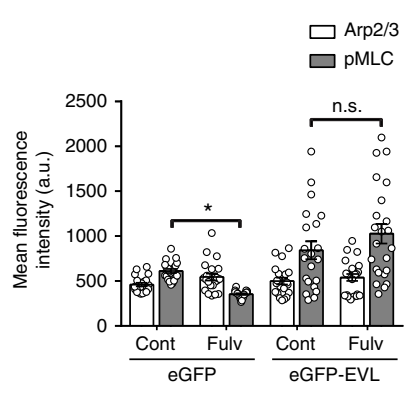

e
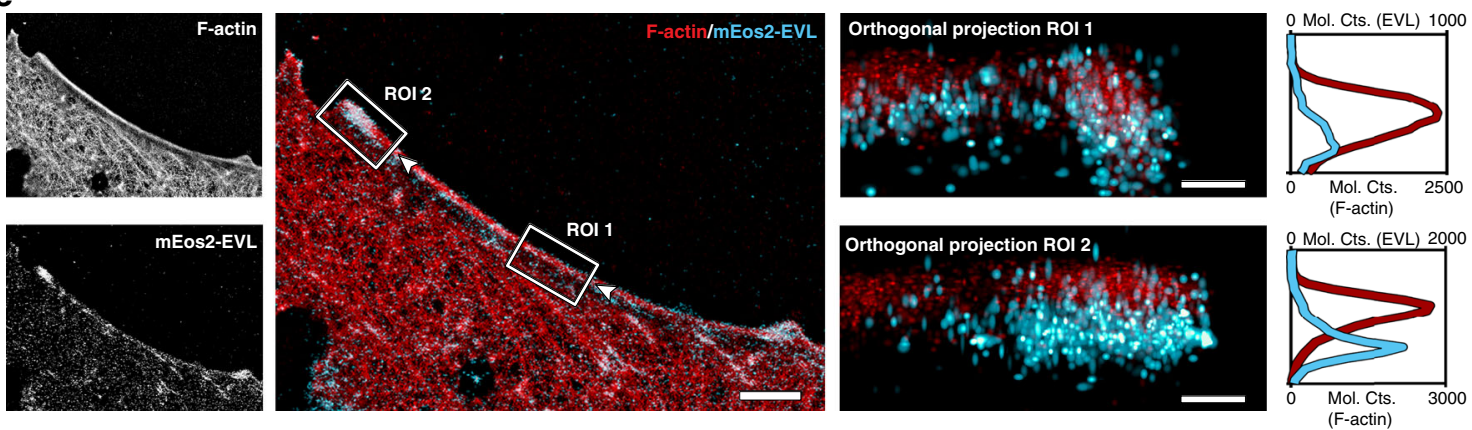

'

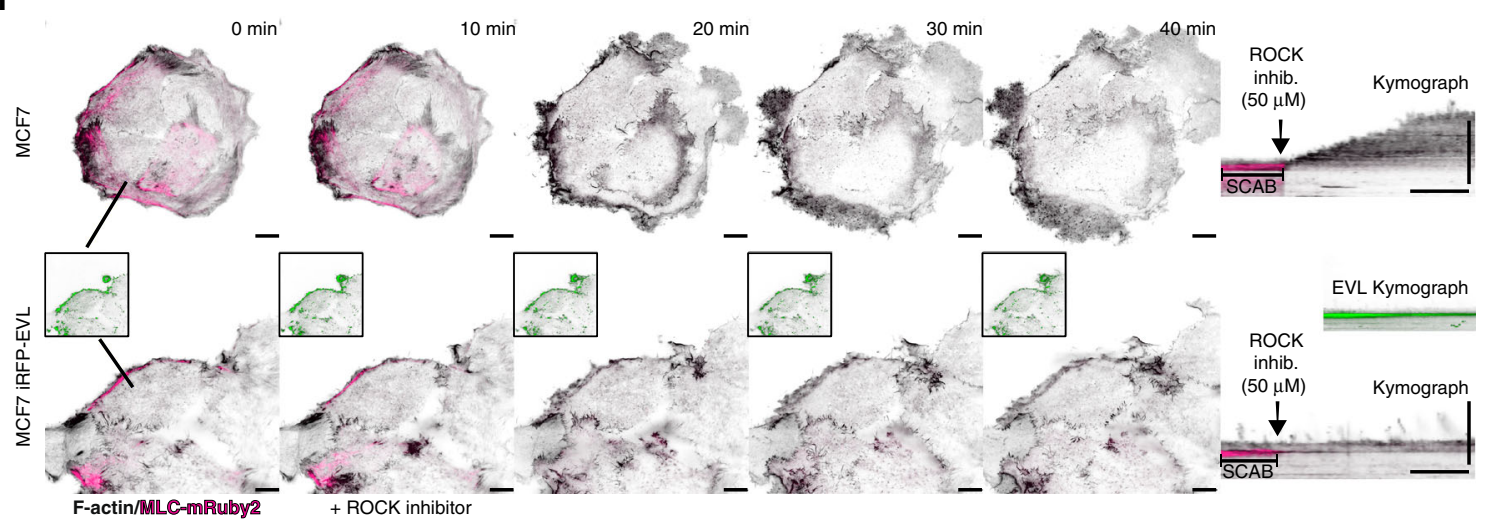


LII measurement: Cancer cell invasion was quantified in both TMAs (64 samples from the NCI CDP-BCP-TMA and 180 samples from the Cedars-Sinai LumB TMA) by employing the NND approach. Cancer cells were identified using a binary mask generated by thresholding the fluorescence signal of the cytokeratin stain. Using a built-in module in Nikon Elements, the distance between the nucleus of each cancer cell and the nucleus of the cell nearest to it was measured. Cells scattered at the periphery of the analyzed region were excluded from our analysis if their NND zone (circular zone with centroid as center and NND as radius) is not fully contained within the analyzed area. LII was calculated as the average NND of all cancer cells within the analyzed tumor area.

Three-dimensional culture system. A tissue culture model system was designed to quantitate 3D invasion of cells with low invasive capacity; the configuration of this system allowed reliable monitoring of invading cells over a relatively long period of time ( $\geq 2$ days). The system was setup in 8-well glass-bottom chamber slides (Labtek), where cancer cells were embedded in a central 3D matrix and allowed to invade into an encapsulating outer 3D matrix. Prior to utilization, the bottom coverslips of the chamber slides were silanized using $2 \%$ aminopropyltrimethoxysilane (Acros Organics) in isopropanol for $10 \mathrm{~min}$ at room temperature, followed by repeated water washes and drying at $37^{\circ} \mathrm{C}$. Chamber slides were then treated with $1 \%$ glutaraldehyde for $20 \mathrm{~min}$ at room temperature, followed by water washes and drying at $37^{\circ} \mathrm{C}$, and finally sterilization under ultraviolet light for $15 \mathrm{~min}$. After corresponding pretreatments, $1 \times 10^{5}$ cells per well were resuspended in $10 \mu \mathrm{L}$ of $1 \mathrm{mg} / \mathrm{mL}$ type-I collagen containing $1.25 \mathrm{mg} / \mathrm{mL}$ polyethylene glycol diglycidyl ether (Fisher). Cell suspension was added at the center of the chamber wells and allowed to polymerize in the tissue culture incubator. The outer matrix, prepared similarly to the collagen mixture described above with the addition of $20 \mathrm{nM}$ human recombinant IGF1, was added to encapsulate the central matrix; after polymerization, the setup was overlaid with growth media containing the corresponding treatments. Cells were allowed to invade into the outer matrix for $48 \mathrm{~h}$ (with one media change at $24 \mathrm{~h}$ ), and then they were fixed and stained with Hoechst 33342 (Thermo Scientific) for $24 \mathrm{~h}$ at $4{ }^{\circ} \mathrm{C}$. Confocal z-series of Hoechst and differential interference contrast (DIC) were acquired at $1.5 \mu \mathrm{m}$ z-steps on a Nikon Ti-E inverted microscope equipped with a $\times 20$ Plan Apo 0.75NA Nikon objective, Photometrics CoolSNAP MYO CCD camera, motorized stage, and a Crest XLIGHT spinning-disk confocal system. At each z-step, large-image stitching was used to generate a composite image of the entire area including the central matrix and the invaded cells. A binary mask was generated from the maximum intensity projection image of the $z$-series. A region of interest (ROI) was created by excluding the central area (determined using the overlaid DIC image) and the number of invading cells in the ROI was quantitated using the Object Count Nikon Elements module.

Kymography analysis. DIC microscopy time-lapse imaging: Cells were plated at $30 \%$ confluence on glass-bottom 6-well plate (MatTek) and allowed to adhere for $24 \mathrm{~h}$ before experimentation. Cells were treated with E2 $(10 \mathrm{nM})$, tam $(1 \mu \mathrm{M})$, or fulv $(100 \mathrm{nM})$ in complete growth media, which were replaced every $24 \mathrm{~h}$. Cells were imaged $72 \mathrm{~h}$ after plating at 1 frame/s for $45 \mathrm{~min}$ using DIC microscopy. Images were acquired with a $\times 60$ Plan Apo 1.40 NA Nikon objective on a Nikon Ti-E inverted microscope equipped with a Hamamatsu ORCA-Flash 4.0 V2 cMOS camera, environmental chamber, motorized stage, and maintained in focus using Nikon Perfect Focus System.

Kymography analysis of membrane ruffles: A kymograph was registered along the axis of ruffling at the site of highest membrane dynamics (as determined by minimum intensity projections). On the generated kymograph, membrane ruffles were identified as areas of high contrast. For each kymograph, two parameters of membrane ruffles were analyzed: frequency and speed. Frequency was determined by quantitating ruffle number (areas of high contrast) in each kymograph. Speed was determined as the distance traveled by a ruffle/time.

Immunofluorescence staining. Cells were fixed with $4 \%$ paraformaldehyde (PFA) (Electron Microscopy Sciences) for $10 \mathrm{~min}$ at $37^{\circ} \mathrm{C}$. PFA was quenched with $0.1 \mathrm{M}$ glycine and cells were permeabilized with $0.1 \%$ Triton X-100, both for $10 \mathrm{~min}$ at room temperature. The actin cytoskeleton was stained with Alexa Fluor-conjugated phalloidin (Alexa Fluor 568-Phalloidin or Alexa Fluor 647-Phalloidin; Thermo Fisher) diluted to $2.5 \%$ in phosphate-buffered saline (PBS); nuclei were stained with Hoechst 33342 (Thermo Scientific); pMLC was immunolabeled using human anti-(Thr18/Ser19) pMLC 2 no. 3674 (Cell Signaling) at 1:100 dilution; Arp2/3 was immunolabeled using human anti-Arp2/3 clone no.13C9 (Millipore) at 1:200 dilution; EVL was immunolabeled using human anti-EVL (a gift from Frank Gertler) at 1:50 dilution. Slides were blocked in $1 \% \mathrm{FBS} / 1 \%$ bovine serum albumin (BSA) in PBS; primary and secondary antibodies were diluted in blocking buffer and incubated using standard protocols. Samples were mounted in ProLong Diamond Antifade (Thermo Fisher) and allowed to cure for at least $24 \mathrm{~h}$ before imaging.

Volumetric analysis. Cells were fixed with 4\% PFA (Electron Microscopy Sciences) for $10 \mathrm{~min}$ at $37^{\circ} \mathrm{C}$. PFA was quenched with $0.1 \mathrm{M}$ glycine and cells were permeabilized with $0.1 \%$ Triton $\mathrm{X}-100$, both for $10 \mathrm{~min}$ at room temperature. The actin cytoskeleton was stained with Alexa Fluor-conjugated phalloidin (Alexa Fluor 568-Phalloidin or Alexa Fluor 647-Phalloidin; Thermo Fisher) diluted in PBS; nuclei were stained with Hoechst 33342 (Thermo Scientific). Slides were blocked in $1 \% \mathrm{FBS} / 1 \% \mathrm{BSA}$ in PBS at room temperature for $1 \mathrm{~h}$. pMLC and Arp $2 / 3$ were immunolabeled with primary and secondary antibodies diluted in blocking buffer and incubated using standard protocols. Confocal z-series were acquired at $0.2 \mu \mathrm{m}$ $z$-steps on a Nikon Ti-E inverted microscope equipped with a $\times 100$ Plan Apo TIRF 1.49NA Nikon objective, Photometrics CoolSNAP MYO CCD camera, motorized stage, a Crest XLIGHT spinning-disk confocal system, and a Lumencor SPECTRA $\mathrm{X}$ light source. Mean fluorescence intensity of pMLC and Arp2/3 at the leading edge was determined within a $3 \mathrm{D}$ volumetric binary mask, generated using the fluorescent signal of the actin stain. Nikon Elements 3D Object Module was used to construct the $3 \mathrm{D}$ binary mask from a series of $2 \mathrm{D}$ masks generated at each z-step in the actin channel. Importantly, to limit the analysis to the leading edge actin, the masks were generated within an ROI that was restricted to a 3- $\mu \mathrm{m}$-wide band juxtaposed to the membrane.

TIRFM time-lapse imaging. Cells expressing Lifeact-iRFP670 and MLC-mRuby2 were used in this experiment to analyze actin dynamics during protrusion at the leading edge. Cells were plated as described above and ROCK inhibitor was added at indicated concentrations to dissolve SCABs. Cells were imaged using TIRF illumination with a $\times 100$ Plan Apo TIRF 1.49NA objective on a Nikon Ti-E inverted microscope equipped with a Hamamatsu ORCA-Flash 4.0 V2 cMOS camera, Photometrics Evolve 512 EMCCD camera. Images were acquired on a 20-s interval for indicated duration. For EVL overexpression time-lapse experiments, cells expressing Lifeact-mEGFP and MLC-mRuby2, with or without iRFP670-EVL, were co-cultured and imaged as described above.

Kymography analysis of actin dynamics: Similar approach as above was used to register the kymographs, with the exception that maximum intensity projection of the fluorescent images from the entire time series was used to guide the positioning of the kymograph line.

Differential gene expression analysis of actin cytoskeletal regulators. GO Consortium search engine was used, with the search term "cytoskeleton," to

Fig. 5 EVL promotes ER-mediated actin remodeling. a Maximum intensity projections of z-series of control and E2-treated LKO and EVL KD MCF7 cells. Scale bar is $10 \mu \mathrm{m}$. b Quantification of leading edge Arp2/3 and pMLC. Data from three independent experiments ( $n=21$ cells per group; mean \pm s.e.m.). Three-way ANOVA shows significant differences between LKO and EVL KD groups at $p=0.08(\mathrm{df}=1)$. Two-way ANOVA shows significant difference in pMLC levels at $p<0.05$ between control and E2-treated cells in the LKO group, but not in the EVL KD group. No significant differences in Arp2/3 levels were observed at $p<0.05 .{ }^{\star \star} p<0.01$. c Maximum intensity projections of $z$-series of control and fulv-treated eGFP and eGFP-EVL MCF7 cells. Scale bar is $10 \mu \mathrm{m}$. Scale bar is $5 \mu \mathrm{m}$ for inset showing eGFP-EVL and pMLC. Larger fields of view from the same group are given in Supplementary Fig. $5 \mathrm{~b}$.

d Quantification of leading edge Arp2/3 and pMLC. Data from three independent experiments ( $n=21$ cells per group; mean \pm s.e.m.). Three-way ANOVA shows significant differences between eGFP and eGFP-EVL groups at $p<0.0001(\mathrm{df}=1)$. Two-way ANOVA shows significant difference in $p M L C$ levels at $p<0.05$ between control and fulv-treated cells in the eGFP group, but not in the eGFP-EVL group. No significant differences in Arp2/3 levels were observed at $p<0.05$. ${ }^{*} p<0.05$. e Analysis of EVL localization at SCABs by iPALM in MCF7 cells expressing mEos2-EVL. Left panels show single and merged images of F-actin staining and mEos2-EVL; boxes indicate ROls. Arrows indicate en face orientation of orthogonal projections. Orthogonal projections of ROIs are shown with corresponding histogram highlighting z-localization of F-actin (red) and mEos2-EVL (cyan); horizontal axes represent molecular counts (mol. cts.) for mEos2-EVL (top) and F-actin (bottom). Merge image scale bar is $5 \mu \mathrm{m}$. Orthogonal projection scale bar is $150 \mathrm{~nm}$. f Leading edge kymography in control and iRFP670-EVL (green) expressing MCF7 cells (top and bottom rows, respectively), with eGFP-Lifeact (black) and MLC-mRuby2 (magenta), before and after ROCK inhibitor ( $50 \mu \mathrm{M})$ treatment (Supplementary Movie 8). Left panels are images from time-lapse series before and after treatment. Scale bar is $10 \mu \mathrm{m}$. Line shows leading edge location at which kymographs were registered. Inset shows EVL channel separately. Right panel shows kymograph. Vertical scale bar is $10 \mu \mathrm{m}$. Horizontal scale bar is $10 \mathrm{~min}$. n.s. nonsignificant 
generate a list of genes associated with the cytoskeleton. The initial list of $1000+$ genes retuned in the GO search (www.geneotology.org) was further refined by selecting only cytoskeletal regulators that directly interact with actin, as determined by hand for each gene from an extensive literature review; this process generated a shorter list of 285 actin cytoskeletal regulators (Supplementary Data 1). Using public datasets available on Oncomine (www.oncomine.org), differential expression in ER+ breast cancer of the genes in our curated gene set was analyzed. The following criteria for inclusion of studies were applied: (1) studies must have included clinical samples from patients (studies with only cell line data were excluded); (2) studies must have included both ER + and ER - tumors, with a minimum of ten samples per group; and (3) microarray chips used in the studies must have had probes representing a minimum of $95 \%$ of the genes on our curated gene set. Based on these criteria, we selected 12 out of the 56 studies available on Oncomine. Data were presented in volcano plots to visualize both fold change and significance, concomitantly. Our thresholds for identifying a gene as a positive "hit" were twofold change in expression (in either positive or negative direction) and $p \leq 0.05$.

a

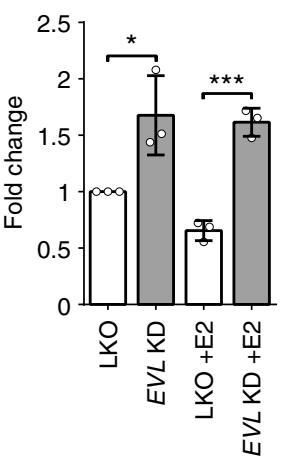

f

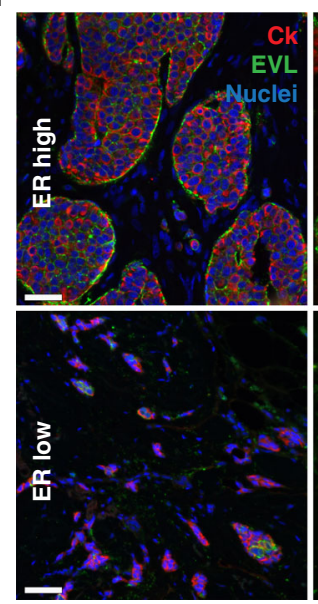

i

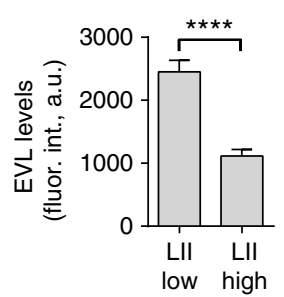

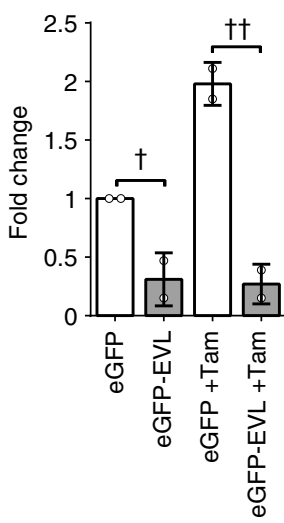

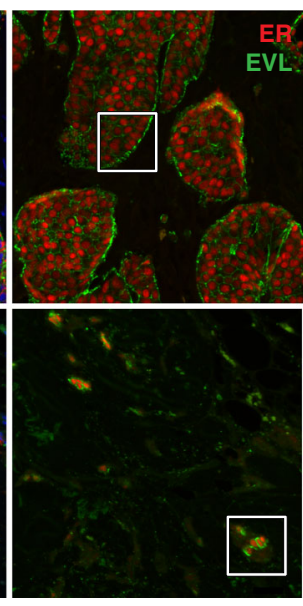

j

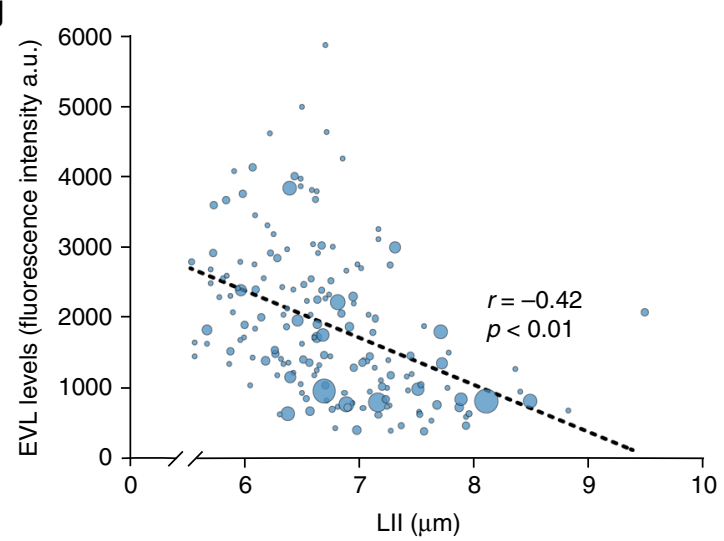

RNA-seq analysis. We analyzed mRNA expression in breast cancer in TCGA breast cancer RNA-seq dataset. RNASeq Version 2 level 3 data were downloaded from TCGA breast cancer; 1062 breast tumors and 113 normal tissue samples were included in the analysis. Tumor samples were classified as ER+ or ER- based on the ER status indicated in the accompanying clinical report. Fold change was computed manually using $\mathrm{R}$ as well as via bioconductor package, DESeq2. Raw counts from level 3 data, which includes non-integer numbers, were rounded off for analysis in DESeq2, which requires integer-based read counts. $P$ value was calculated using paired $t$ test for manual calculation and Benjamini-Hochberg method was used for multiple test corrections. The bioconductor package, pheatmap3, was used to plot heat maps using row scaling on gene counts and clustering was enabled for gene clustering

ChIP assay. Experiments were performed as described previously ${ }^{42}$. Briefly, prior to the assay, cells were hormone-starved for 3 days in phenol-free DMEM base media supplemented with $10 \%$ charcoal-stripped FBS. Starved cells were treated for e
C
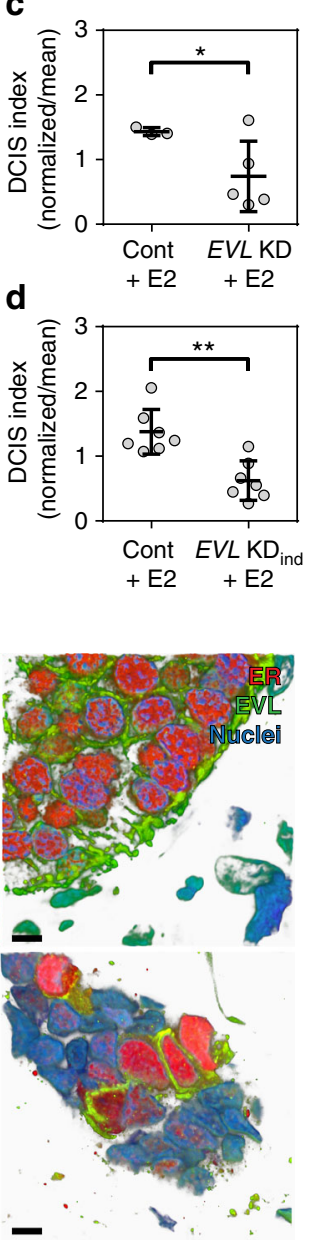

g

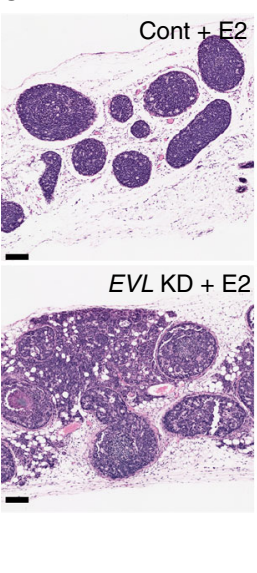

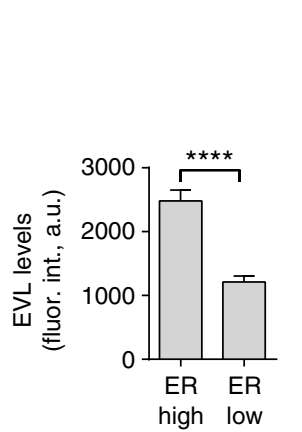
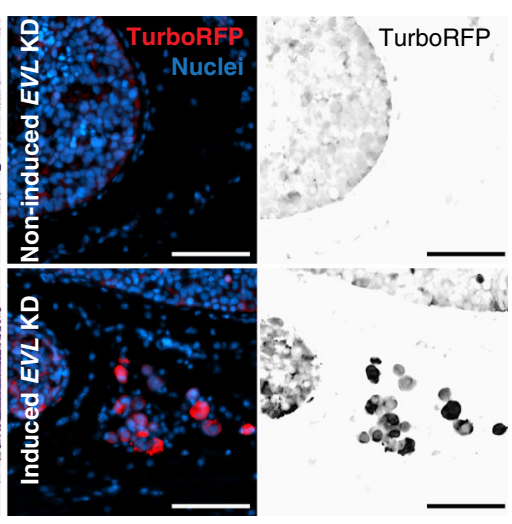

h

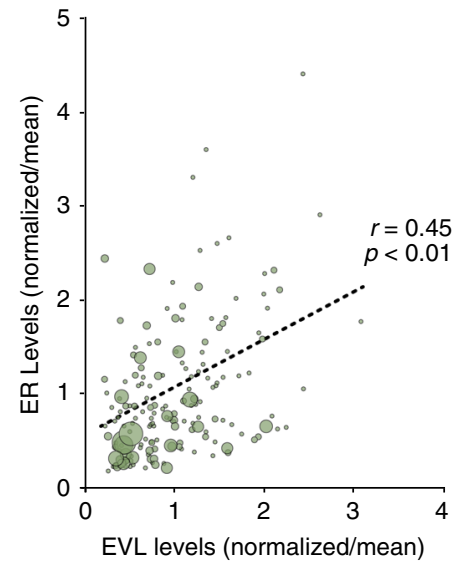

k

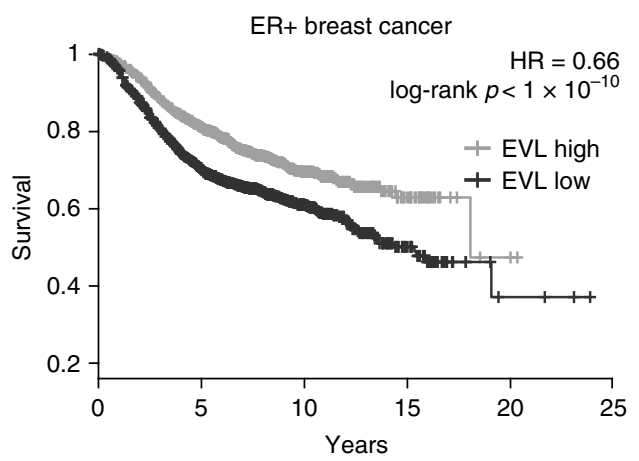


45 min with E2 (10 nM). For ChIP, chromatin was cross-linked with 1\% PFA (Electron Microscopy Sciences) for $8 \mathrm{~min}$ at room temperature. PFA was then quenched using $125 \mathrm{mM}$ glycine and $5 \mathrm{mg} / \mathrm{mL}$ BSA. Cells were scraped with PBS and cellular pellets were isolated by centrifugation at $2000 \mathrm{rpm}$ for $5 \mathrm{~min}$ at $4{ }^{\circ} \mathrm{C}$. Pellets were resuspended in lysis buffer (1\% sodium dodecyl sulfate (SDS), $10 \mathrm{mM}$ EDTA and $50 \mathrm{mM}$ Tris, $\mathrm{pH} 8.0$ ) and sonicated using an E210 instrument (Covaris) to an average size of $350 \mathrm{bp}$ according to the manufacturer's protocol. After clearing by centrifugation at $15,000 \mathrm{rpm}$ for $15 \mathrm{~min}, 5 \mu \mathrm{l}$ of sample was collected as input before immunoprecipitation, and the rest was diluted fivefold in dilution buffer (20 mM Tris, pH 8.0, $150 \mathrm{mM} \mathrm{NaCl}, 2 \mathrm{mM}$ EDTA, and 1\% Triton X-100). Chromatin complexes were immunoprecipitated by an overnight incubation at $4{ }^{\circ} \mathrm{C}$ with $2 \mu \mathrm{g}$ anti-ER antibodies (Santa Cruz, HC-20 and Thermo Fisher, AB-10), followed by a $45 \mathrm{~min}$ incubation with $40 \mu \mathrm{l}$ 1:1 mix of protein A and G Dynabeads (Invitrogen). Beads were then washed four times with RIPA lysis buffer $(50 \mathrm{mM}$ HEPES, $1 \mathrm{mM}$ EDTA, $0.7 \%$ deoxycholate, $1 \% \mathrm{NP}-40$, and $0.5 \mathrm{M} \mathrm{LiCl}$ ) and twice with Tris-EDTA (TE) buffer. The protein-DNA complexes were eluted in 1\% SDS, $0.1 \mathrm{M}$ sodium bicarbonate buffer heated at $65^{\circ} \mathrm{C}$ for $6 \mathrm{~h}$ to reverse the PFA crosslinking. DNA was isolated using PCR Cleanup Kit (Qiagen) and quantitated using Quant-IT as per the manufacturer's instructions (Thermo Scientific). DNA fragments were analyzed by qPCR using SYBR Green Master Mix (Kapa Biosciences) on an ABI 7300 instrument (Applied Biosystems). For ChIP-seq, purified DNA was then end repaired and ligated to adapter oligos (Illumina) and amplified. Sequencing was performed at the Center for Cancer Computational Biology at Dana Farber Cancer Institute using an Illumina HiSeq2000 instrument. Reads were mapped to the human genome using STAR and peaks indicating ER binding were called comparing ChIP and corresponding Chromatin Input libraries using MACS2.1 and a threshold of $p<10^{-5}$. ChIP-seq SPMR (signal per million reads) traces were generated using MACS2.1 followed by conversion to BigWig format using the bedGraphToBigWig tool. High confidence binding cutoff was arbitrarily chosen at $q<10^{-200}$ and presence in both ChIP-seq replicates corresponding to approximately $10 \%$ of the entire dataset.

Quantitative real-time PCR. Cells were treated for $24 \mathrm{~h}$ with E2 $(10 \mathrm{nM})$, tam $(1 \mu \mathrm{m})$, or fulv $(100 \mathrm{nM})$; E2-treated cells were hormone-starved for $24 \mathrm{~h}$ as described above. Total RNA was isolated using Isolate II RNA Kit (Bioline) and cDNA was then synthesized from $1 \mu \mathrm{g}$ of RNA using XLA Script cDNA Kit (Quanta BioSciences). SYBR Green PCR Mix (Apex) was used for Real Time qPCR on the ABI Fast 7500 system. Samples were run in triplicates in each experiment and relative mRNA levels were normalized to GAPDH (glyceraldehyde 3phosphate dehydrogenase). qPCR primer sequences are found in Supplementary Table 9 .

Quantification of EVL protein by immunofluorescence. Cells were fixed and stained as described above and imaged using a $\times 20$ Plan Apo 0.75NA Nikon objective on a Nikon Ti-E inverted microscope equipped with a Hamamatsu ORCA-Flash 4.0 V2 cMOS camera, motorized stage and maintained in focus using Nikon Perfect Focus System. For each drug treatment, two large composite images of $8 \times 8$ fields of view were generated by digital stitching using Nikon Elements. To quantify EVL levels, a binary mask was generated against F-actin staining using Nikon Threshold Module and EVL signal was quantified within this mask.

DCIS mouse model. A previously described protocol of intraductal injection was followed with modifications ${ }^{43,44}$. In brief, 8 -week-old female nonobese diabetic/ severe combined immunodeficiency mice were ovariectomized and implanted with a sub-dermal 90-day release $0.72 \mathrm{mg}$ E2 pellets (Innovative Research of America). Two days after implantation, $1 \times 10^{4}$ cells suspended in $3 \mu \mathrm{L}$ growth media were injected into the primary mammary duct through a cleaved nipple, using a
Hamilton syringe. For the first experiment, we used MCF7 cells expressing shRNA targeting EVL and control cells expressing the LKO vector control; for the second experiment, we used cells expressing an inducible form of shRNA targeting $E V L$ and TRIPZ vector control. After 6-8 weeks, tumors were harvested, fixed in $4 \%$ PFA (Electron Microscopy Sciences), and processed for tissue staining. For experiments with cells expressing an inducible TRIPZ constructs, doxycycline was administered in the drinking water at a concentration of $2 \mathrm{mg} / \mathrm{mL}$ for 2 weeks before tumor harvesting. All experiments were performed according to the guidelines of the IACUC committee of the University of Arizona.

Analysis of DCIS index. Quantification of DCIS index: Large composite images of the entire mid-sections from each tumor sample were acquired using a $\times 4$ Plan Fluor 0.13 NA Nikon objective on a TI-Eclipse TE2000-U inverted microscope equipped with a Nikon DS-Fi2 color camera and motorized Prior stage. DCIS structures were identified as intact non-disseminated tumor structures and were counted in the composite images. In addition, tumor area, which encompasses the whole tumor region, and cellular area, which consists of only the area with cancer cells (i.e., excluding the stroma), were measured; the cellular coefficient was calculated as cellular area/tumor area. The DCIS index was calculated as the product of the number of DCIS structures multiplied by the cellular coefficient (to correct for differences in cellularity among tumors). The DCIS index was then normalized over the tumor area (to correct for differences in tumor size).

\section{Interferometric photoactivated localization microscopy. MCF7 cells, trans-} duced with pCMV-mEos2-EVL-IRES-BlastR lentivirus, were cultured for $24 \mathrm{~h}$ on 25-mm-diameter coverslips containing gold nanoparticles (Nanopartz) that serve as iPALM calibration standards and drift correction fiducial markers ${ }^{38,45}$. Cells were fixed using 2\% PFA in PEM buffer (80 mM PIPES, 5 mM EGTA, $2 \mathrm{mM}$ $\mathrm{MgCl}_{2}, \mathrm{pH}$ 6.8) and the actin cytoskeleton was stained using phalloidin conjugated to Alexa Fluor 647 (Thermo Fisher Scientific) at a concentration of $2.5 \%(5 \mathrm{U} / \mathrm{mL})$. Immediately following labeling, samples were mounted in STORM buffer $(100 \mathrm{mM}$ MEA, $0.4 \mathrm{mg} / \mathrm{mL}$ glucose oxidase, and $24 \mu \mathrm{g} / \mathrm{mL}$ catalase from Sigma), $10 \%$ glucose, $10 \mathrm{mM} \mathrm{NaCl}$, and $50 \mathrm{mM}$ Tris- $\mathrm{HCl}$, pH 8.0. A second $18 \mathrm{~mm}$ diameter coverslip was adhered to the top of the sample and sealed to create an air-tight imaging volume, permitting sequential 3D super-resolution imaging of Alexa Fluor 647-Phalloidin via STORM and mEos2-EVL via PALM. Samples were imaged using interferometric photoactivation and localization microscopy, as previously described. Briefly, images were acquired via two $\times 60$ TIRF 1.49NA objectives (Nikon), with signals from both combined in a custom three-way beamsplitter (Rocky Mountain Instruments), and imaged in three phase channels on EMCCD cameras (iXon DU-897, Andor Technology). Alexa Fluor 647-conjugated phalloidin was imaged first, using $642 \mathrm{~nm}$ laser excitation (OptoEngine LLC) operating at ca. $5 \mathrm{~kW} / \mathrm{cm}^{2}$ at the sample, with camera exposure of $40 \mathrm{~ms}$ to acquire 60,000 raw frames. mEos2-EVL was imaged using $561 \mathrm{~nm}$ laser excitation (OptoEngine LLC) at ca. $2.5 \mathrm{~kW} / \mathrm{cm}^{2}$, with $2-10 \mathrm{~W} / \mathrm{cm}^{2}$ illumination at $405 \mathrm{~nm}$ (Coherent) for photoconversion of mEos2, and $50 \mathrm{~ms}$ camera exposure to acquire 50,000 raw images. Raw images were then subjected to iPALM localization, final image reconstruction, multichannel alignment, and analysis using PeakSelector software ${ }^{46}$. Final en face and orthogonal ROIs are displayed as cumulative intensity projections of rendered localizations.

Laser scanning confocal microscopy. Cells were embedded in a 3D type-I collagen matrix in 8 -well glass-bottom chamber slides as previously described in Three-Dimensional Culture System methods. Cells were imaged using a $\times 60$ Plan Apo 1.42 NA Olympus Objective on an Olympus Fluoview1200 laser scanning confocal microscope. Images are maximum intensity projections of confocal z-series.

Fig. 6 EVL suppresses invasive activity of breast cancer cells and is associated with low dissemination in breast tumors. a Fold change in invasion of LKO and EVL KD control and E2-treated MCF7 cells; mean \pm s.d.; ${ }^{\star} p=0.02,{ }^{\star \star \star} p=0.004$ (unpaired $t$ test). Data are from three independent experiments. b Fold change in invasion of control and tam-treated eGFP and eGFP-EVL MCF7 cells; mean \pm s.d.; $\dagger^{\dagger} p=0.04, \dagger p=0.01$ (unpaired $t$ test). Data are from three independent experiments. c DCIS index in LKO and constitutive EVL KD MCF7 xenograft tumors treated with E2 ( $n=3$ mice in LKO and $n=5$ in $E V L$ KD group; mean \pm s.d. ${ }^{*} p=0.04$ ) (Welch's $t$ test). $\mathbf{d}$ DCIS index in control and inducible EVL KD tumors $\left(n=7\right.$ mice per group; mean \pm s.d.; $\left.{ }^{\star *} p=0.001\right)$ (Welch's $t$ test). e Representative H\&E stains (left), scale bar is $500 \mu \mathrm{m}$; and doxycycline induction (right), scale bar is $100 \mu \mathrm{m}$. f Representative luminal B breast tumors with high (top) or low (bottom) ER expression (Cedars-Sinai LumB TMA). Left panels show merged images of cytokeratin (red) and EVL (green) immunolabeling and middle panels of ER (red) and EVL (green) immunolabeling. Images are maximum intensity projections of z-series. Scale bar is $50 \mu \mathrm{m}$. Right panels are 3D reconstructions of boxed areas, imaged at higher resolution. Scale bar is $5 \mu \mathrm{m}$. $\mathbf{g}$ Quantification of EVL levels in ER low (1st quartile) and ER high (3rd quartile) tumors; a.u. = arbitrary units; mean \pm s.e.m.; ${ }^{\star \star \star \star} p<0.0001$ (Welch's $t$ test). $\mathbf{h}$ Scatter plot of ER vs. EVL levels; bubble area is proportional to the number of positive lymph nodes in the corresponding patient; $r$ is Pearson's correlation coefficient; correlation is significant at $p<0.01 ;$ a.u. = arbitrary units. i Quantification of EVL levels in LII low $(\leq 7 \mu \mathrm{m})$ and LII high $(>9 \mu \mathrm{m})$ tumors by mean fluorescence intensity; a.u. $=$ arbitrary units; mean \pm s.e.m. ${ }^{\star \star \star \star} p<0.0001$ (Welch's $t$ test). $\mathbf{j}$ Scatter plot of EVL levels and LII; bubble area is proportional to the number of positive lymph nodes in the corresponding patient; $r$ is Pearson's correlation coefficient; correlation is significant at $p<0.01$. k Survival of ER+ breast cancer patients clustered by EVL expression (split at median). Data from KM plotter (Affy ID =217838_s_at/gene symbol =EVL; ER status derived from gene expression). Hazard ratio $(H R)$ and $p$ value shown in inset (log-rank test); 1541 patients in the EVL high group, 1541 patients in the EVL low group 
CRISPR/Cas9 knock-in. Cells were edited using the CRISPR/Cas9 as previously described ${ }^{47}$. Briefly, the three following constructs were co-transfected to edit endogenous EVL: (1) a plasmid coding for the SpCas9 endonuclease; (2) a template plasmid coding for the sequence of eGFP and a flexible linker with an amino acid sequence of GGSGGSGGS flanked upstream and downstream by $~ 800$ bp of the ATG codon of $E V L$; and (3) a linear double-stranded DNA product obtained by PCR amplification and purification coding for the U6 promoter, the genomic RNA (gRNA) targeting the ATG region of the EVL gene and the tracrRNA recognized by Cas9. The gRNA sequence was ACTTTTCAGCCATGGCCACA, the underlined nucleotides representing the start codon of the $E \overline{V L}$ gene. Edited cells (eGFP-
$E V L^{\text {edited }}$ ) were generated by transfection, at $70 \%$ confluence, with $600 \mathrm{ng}$ of each of the above-mentioned constructs using Fugene HD in OptiMEM. Edited cells were enriched by fluorescence-activated cell sorting on a FACSAria II and efficient editing of $E V L$ was verified by fluorescence microscopy and validated by genomic PCR

Western blot analysis. Cells were scraped on ice in lysis buffer (10\% glycerol, $1 \%$ IGEPAL, $50 \mathrm{mM}$ Tris, pH 7.5, $200 \mathrm{mM} \mathrm{NaCl}, 2 \mathrm{mM} \mathrm{MgCl}$, protease inhibitor cocktail from Proteomics, M250-1ML, and phosphatase inhibitor cocktail from a

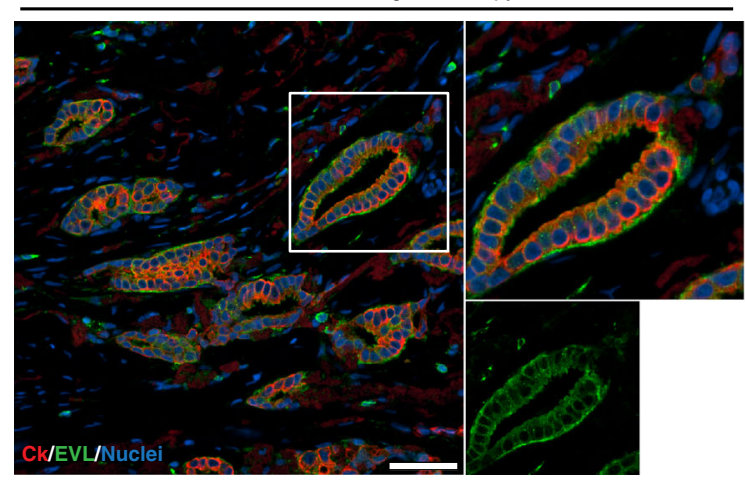

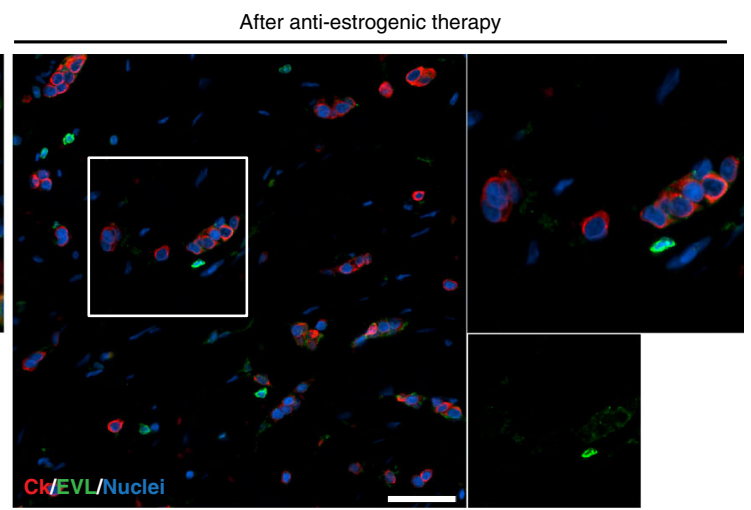

b
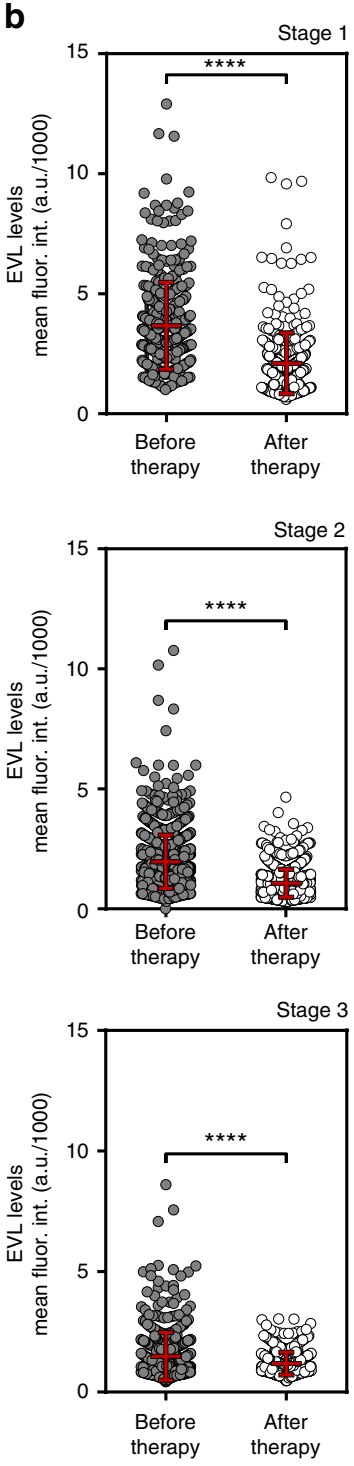

C
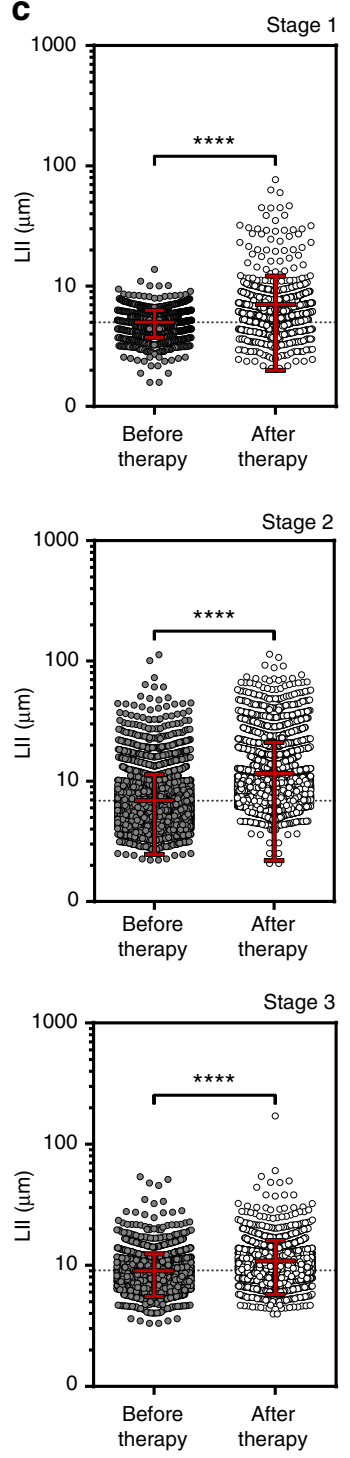
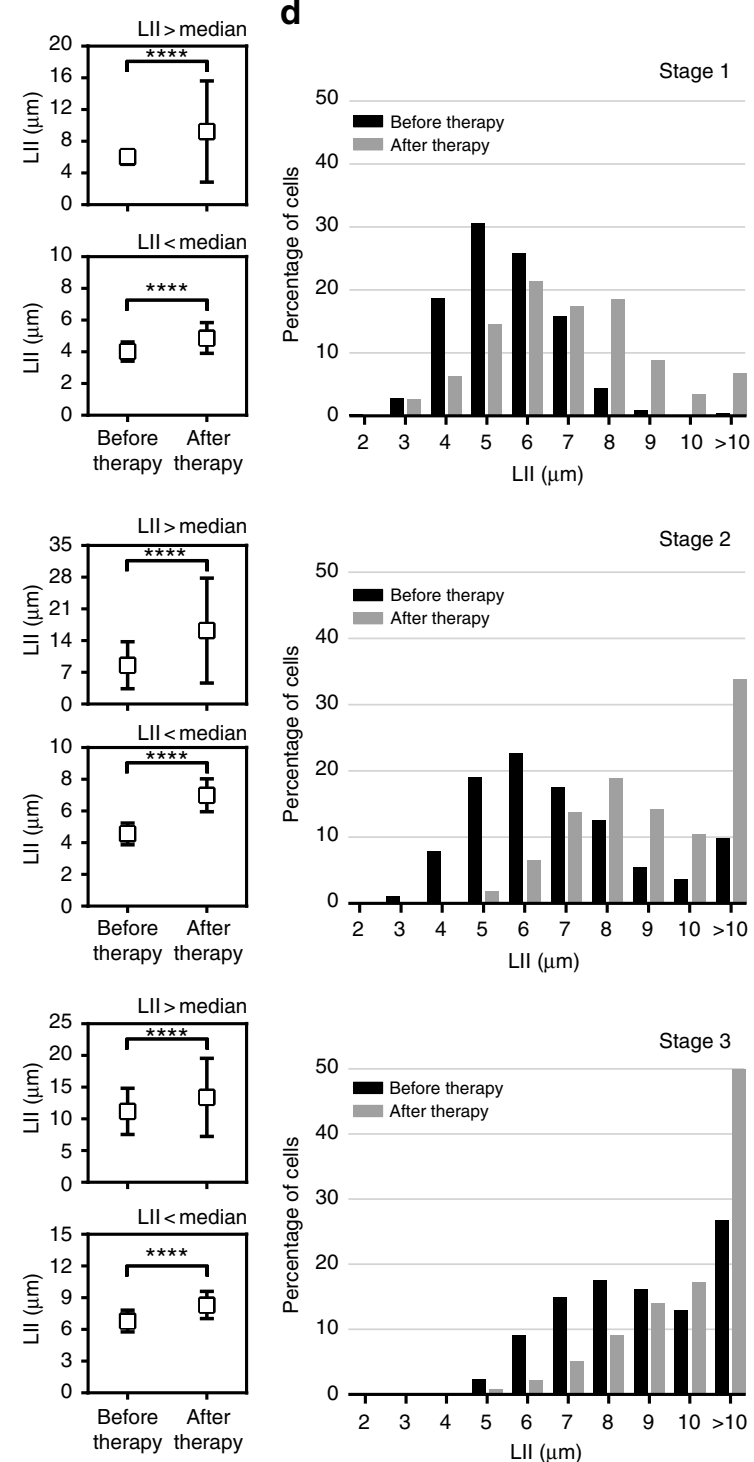

d
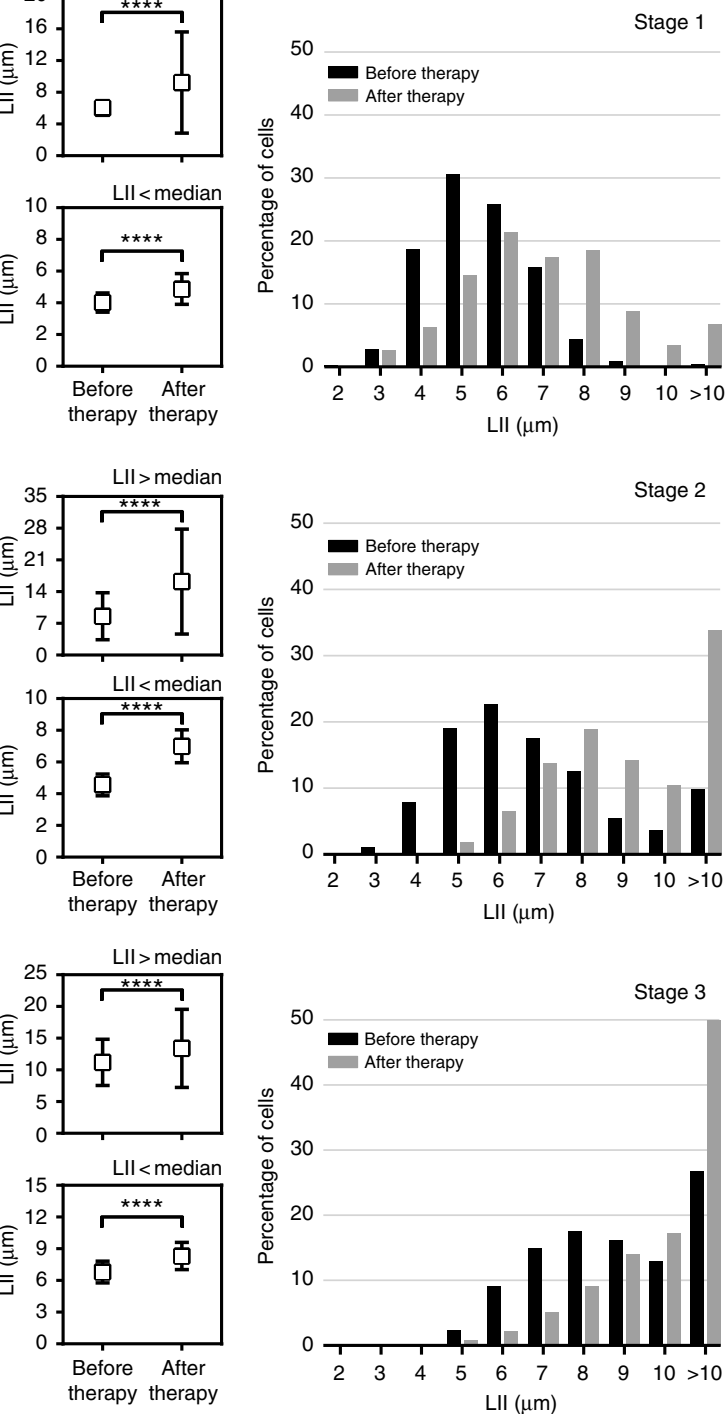
Fig. 7 Anti-estrogenic hormone therapy is associated with decreased EVL expression and increased local invasion in tumors post-treatment.

a Representative images from the same ER+ breast tumor before (left panel) and after (right panel) neoadjuvant hormone therapy. Human cytokeratin is shown in red, EVL in green, and nuclei in blue. Scale bar is $100 \mu \mathrm{m}$. In each panel, top-right inset shows magnification of the boxed area and bottom-right inset shows the EVL channel separately. $\mathbf{b}$ Quantification of EVL levels before and after hormone therapy. Scatter plots show the full range of cells analyzed within each tumor set: stage $1, n=425$ cells before and $n=422$ cells after therapy; stage $2, n=1256$ cells before and $n=1185$ cells after therapy; and stage $3, n=585$ before and $n=501$ after therapy. Red lines represent mean \pm s.d. ${ }^{\star \star \star \star} p<0.0001$ (Welch's $t$ test). c Quantification of local invasion index (LII) before and after therapy. Scatter plots show the full range of cells analyzed within each tumor set: stage $1, n=1259$ cells before and $n=1335$ cells after therapy; stage 2,n=7113 cells before and $n=3987$ cells after therapy; and stage $3, n=2701$ before and $n=2644$ after therapy. Red lines represent mean \pm s.d. ${ }^{\star \star \star \star} p<0.0001$ (Welch's $t$ test); right insets show values from each scatter plot segregated into values $>$ median and values $<$ median $\left({ }^{\star \star \star *} p<0.0001 ;\right.$ Welch's $t$ test). d Histograms showing percentage of cells within different LII bins, before and after therapy

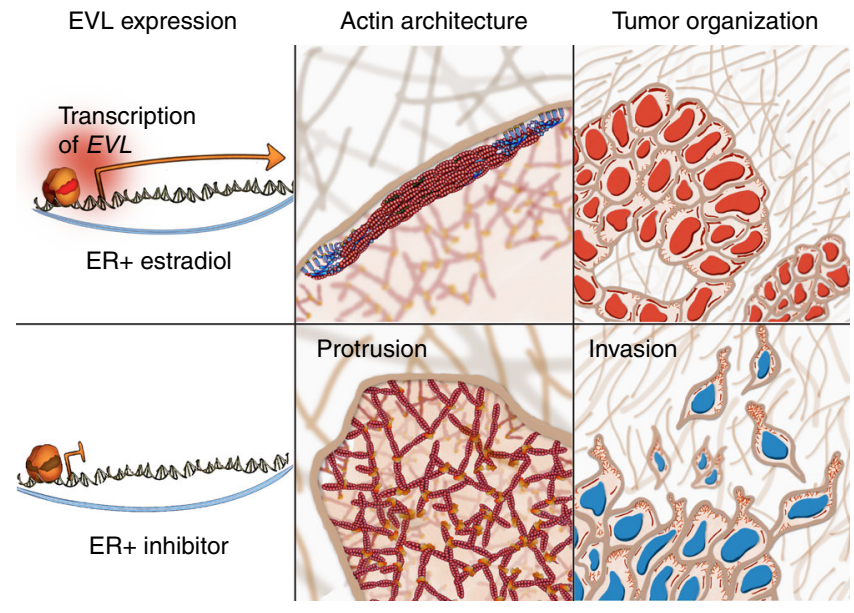

Fig. 8 Model: Suppression of breast cancer cell invasion by ER. ER activates EVL transcription. EVL inhibits invasion by generating SCABs (suppressive cortical actin bundles), which suppress protrusive activity. ER inhibition leads to decrease in EVL levels and increase in protrusive activity and invasion. Red nuclei represent ER positivity

Boston BioProducts (BP479)). Lysates were incubated on ice for 15 min followed by clarification at $14,000 \mathrm{rpm}$ for $15 \mathrm{~min}$ at $4^{\circ} \mathrm{C}$. Protein concentrations were measured using the Bradford assay (Thermo) and $25 \mu \mathrm{g}$ total protein from each sample were resolved by SDS-polyacrylamide gel electrophoresis on $10 \%$ polyacrylamide gels. For film, samples were transferred onto polyvinylidene difluoride membranes (Millipore), which were blocked overnight in $5 \% \mathrm{BSA}$ at $4{ }^{\circ} \mathrm{C}$, and then probed with polyclonal rabbit anti-EVL antibody (a gift from Frank Gertler) at 1:2000 dilution, overnight at $4{ }^{\circ} \mathrm{C}$, followed by standard western blotting protocol. Goat anti-rabbit or anti-mouse horseradish peroxidase secondary antibodies (Thermo Fisher) used with Enhanced Chemi-Luminescence (ECL) (BioRad Clarity Western ECL Substrate, 170-5061). To re-probe following ECL western blotting, antibodies were stripped in $0.2 \mathrm{M} \mathrm{NaOH}$ for $15 \mathrm{~min}$ at room temperature, and membranes were re-probed for actin, as a loading control, using monoclonal mouse anti-actin antibody (Abcam, ab3280, 1:1000) and developed following the same western blot protocol. For Licor, samples were transferred onto nitrocellulose membranes (GE Healthcare), which were blocked for $1 \mathrm{~h}$ in $0.5 \times$ casein (Thermo Fisher), and then probed with polyclonal rabbit anti-EVL antibody (Sigma Prestige HPA018849) at 1:500 dilution, overnight at $4{ }^{\circ} \mathrm{C}$. Near-infrared fluorescent (Licor) secondary antibodies were used for detection. Membranes were scanned and reprobed with mouse anti-actin (ProteinTech 66009-1-Ig, 1:5000) and mouse antiGFP (ProteinTech 50430-2-AP, 1:4000).

Proliferation assay. Cells were plated in a 6-well plate $(25,000$ cells per well) in growth media and allowed to adhere for $24 \mathrm{~h}$. A full media change with drug was performed every day and maintained for $48 \mathrm{~h}$, after which cells were trypsonized and quantified using a hemocytometer.

Statistical analysis. Unless indicated otherwise, statistical summaries are represented as mean \pm s.e.m or mean \pm s.d and statistical significance was determined using a two-tailed $t$ test with Welch's correction. The nonparametric Mann-Whitney test was used in the presence of skewed data. The Pearson's correlation coefficient was estimated to assess the relationship between continuous variables. For ChIP-qPCR experiments, $p$ values were generated by two-way ANOVA (analysis of variance) using the Sidak test for multiple comparison to one control. Survival was estimated using Kaplan-Meier plots. Comparison between survival curves was performed using the log-rank test.
Data availability. Survival data and RNA-seq data in the current study were derived by analysis of The Cancer Genome Atlas, a data repository publicly available at https://portal.gdc.cancer.gov. Microarray data are published and publicly available in the corresponding referenced studies. ChIP-seq data are deposited on GEO repository (GSE116768).

Received: 19 June 2017 Accepted: 4 July 2018

Published online: 30 July 2018

\section{References}

1. Zhang, X. H.-F., Giuliano, M., Trivedi, M. V., Schiff, R. \& Osborne, C. K. Metastasis dormancy in estrogen receptor-positive breast cancer. Clin. Cancer Res. 19, 6389-6397 (2013).

2. Osborne, C. K. \& Schiff, R. Mechanisms of endocrine resistance in breast cancer. Annu. Rev. Med. 62, 233-247 (2011).

3. Collaborative Group on Hormonal Factors in Breast Cancer. Menarche, menopause, and breast cancer risk: individual participant meta-analysis, including 118964 women with breast cancer from 117 epidemiological studies. Lancet Oncol. 13, 1141-1151 (2012).

4. Million Women Study Collaborators. Breast cancer and hormone-replacement therapy in the Million Women Study. Lancet 362, 419-427 (2003).

5. Anderson, G. L. et al. Conjugated equine oestrogen and breast cancer incidence and mortality in postmenopausal women with hysterectomy: extended follow-up of the Women's Health Initiative randomised placebocontrolled trial. Lancet Oncol. 13, 476-486 (2012).

6. Czernichow, C. Traitement hormonal substitutif de la ménopause et risque de cancer du sein. Bull. Cancer 90, 821-831 (2007).

7. Jones, C., Ingram, D. \& Mattes, E. The effect of hormone replacement therapy on prognostic indices in women with breast cancer. Med. J. Aust. 161, 106-110 (1994).

8. Bonnier, P. et al. Impact of menopausal hormone-replacement therapy on clinical and laboratory characteristics of breast cancer. Int. J. Cancer 79, 278-282 (1998).

9. Vidya, R. Data on effect of HRT on breast cancer conflict with other data. BMJ 321, 179-179 (2000).

10. Delgado, R. C. \& Lubian Lopez, D. M. Prognosis of breast cancers detected in women receiving hormone replacement therapy. Maturitas 38, 147-156 (2001).

11. Sacchini, V. et al. Pathologic and biological prognostic factors of breast cancers in short- and long-term hormone replacement therapy users. Ann. Surg. Oncol. 9, 266-271 (2002).

12. Cutuli, B. et al. A French national survey on infiltrating breast cancer: analysis of clinico-pathological features and treatment modalities in 1159 patients. Breast Cancer Res. Treat. 95, 55-64 (2006).

13. Schuetz, F. et al. Reduced incidence of distant metastases and lower mortality in 1072 patients with breast cancer with a history of hormone replacement therapy. Am. J. Obstet. Gynecol. 196, 342.e1-342.e9 (2007).

14. Rauh, C. et al. Hormone therapy and its effect on the prognosis in breast cancer patients. Geburtshilfe Frauenheilkd. 75, 588-596 (2015).

15. Obi, N. et al. Relationship between menopausal hormone therapy and mortality after breast cancer. The MARIE plusstudy, a prospective case cohort. Int. J. Cancer 138, 2098-2108 (2016).

16. Gatrell, A. C., Bailey, T. C., Diggle, P. J. \& Rowlingson, B. S. Spatial point pattern analysis and its application in geographical epidemiology. Trans. Inst. Br. Geogr. 21, 256 (1996).

17. Haque, R. et al. Impact of breast cancer subtypes and treatment on survival: an analysis spanning two decades. Cancer Epidemiol. Biomark. Prev. 21, 1848-1855 (2012).

18. Curtis, C. et al. The genomic and transcriptomic architecture of 2,000 breast tumours reveals novel subgroups. Nature 486, 346-352 (2012). 
19. Platet, N., Cathiard, A. M., Gleizes, M. \& Garcia, M. Estrogens and their receptors in breast cancer progression: a dual role in cancer proliferation and invasion. Crit. Rev. Oncol. Hematol. 51, 55-67 (2004).

20. Gao, Y. et al. Loss of ERa induces amoeboid-like migration of breast cancer cells by downregulating vinculin. Nat. Commun. 8, 14483 (2017).

21. Meyer, A. S. et al. 2D protrusion but not motility predicts growth factorinduced cancer cell migration in 3D collagen. J. Cell Biol. 197, 721-729 (2012).

22. Bravo-Cordero, J. J., Hodgson, L. \& Condeelis, J. Directed cell invasion and migration during metastasis. Curr. Opin. Cell Biol. 24, 277-283 (2012).

23. Lomakin, A. J. et al. Competition for actin between two distinct F-actin networks defines a bistable switch for cell polarization. Nat. Cell Biol. 17, 1435-1445 (2015).

24. Fischer, R. S., Gardel, M., Ma, X., Adelstein, R. S. \& Waterman, C. M. Local cortical tension by myosin II guides 3D endothelial cell branching. Curr. Biol. 19, 260-265 (2009)

25. Vicente-Manzanares, M., Zareno, J., Whitmore, L., Choi, C. K. \& Horwitz, A. F. Regulation of protrusion, adhesion dynamics, and polarity by myosins IIA and IIB in migrating cells. J. Cell. Biol. 176, 573-580 (2007).

26. Mouneimne, G. et al. Differential remodeling of actin cytoskeleton architecture by profilin isoforms leads to distinct effects on cell migration and invasion. Cancer Cell. 22, 615-630 (2012).

27. Bittner, M. Expression project for oncology. International Genomic Consortium GSEE2109 http://www.ncbi.nlm.nih.gov/geo/query/acc.cgi? acc $=$ GSE2109 (2005).

28. Ginestier, C. et al. Prognosis and gene expression profiling of 20q13-amplified breast cancers. Clin. Cancer Res. 12, 4533-4544 (2006).

29. Richardson, A. L. et al. X chromosomal abnormalities in basal-like human breast cancer. Cancer Cell 9, 121-132 (2006).

30. Lu, X. et al. Predicting features of breast cancer with gene expression patterns. Breast Cancer Res. Treat. 108, 191-201 (2008).

31. Korde, L. A. et al. Gene expression pathway analysis to predict response to neoadjuvant docetaxel and capecitabine for breast cancer. Breast Cancer Res. Treat. 119, 685-699 (2010).

32. Glück, S. et al. TP53 genomics predict higher clinical and pathologic tumor response in operable early-stage breast cancer treated with docetaxelcapecitabine \pm trastuzumab. Breast Cancer Res. Treat. 132, 781-791 (2012).

33. Kao, K.-J., Chang, K.-M., Hsu, H.-C. \& Huang, A. T. Correlation of microarray-based breast cancer molecular subtypes and clinical outcomes: implications for treatment optimization. BMC Cancer 11, 143 (2011).

34. Stickeler, E. et al. Basal-like molecular subtype and HER4 up-regulation and response to neoadjuvant chemotherapy in breast cancer. Oncol. Rep. 26, 1037-1045 (2011).

35. Cancer Genome Atlas Network. Comprehensive molecular portraits of human breast tumours. Nature 490, 61-70 (2012).

36. Esserman, L. J. et al. Chemotherapy response and recurrence-free survival in neoadjuvant breast cancer depends on biomarker profiles: results from the ISPY 1 TRIAL (CALGB 150007/150012; ACRIN 6657). Breast Cancer Res. Treat. 132, 1049-1062 (2012).

37. Miyake, T. et al. GSTP1 expression predicts poor pathological complete response to neoadjuvant chemotherapy in ER-negative breast cancer. Cancer Sci. 103, 913-920 (2012)

38. Shtengel, G. et al. Interferometric fluorescent super-resolution microscopy resolves 3D cellular ultrastructure. Proc. Natl. Acad. Sci. USA 106, 3125-3130 (2009).

39. Sflomos, G. et al. A preclinical model for ERa-positive breast cancer points to the epithelial microenvironment as determinant of luminal phenotype and hormone response. Cancer Cell. 29, 407-422 (2016).

40. Tavares, S. et al. Actin stress fiber organization promotes cell stiffening and proliferation of pre-invasive breast cancer cells. Nat. Commun. 8, 15237 (2017).

41. Sweeney, E. E., McDaniel, R. E., Maximov, P. Y., Fan, P. \& Jordan, V. C. Models and mechanisms of acquired antihormone resistance in breast cancer: significant clinical progress despite limitations. Horm. Mol. Biol. Clin. Investig. 9, 143-163 (2012).

42. Carroll, J. S. et al. Chromosome-wide mapping of estrogen receptor binding reveals long-range regulation requiring the forkhead protein FoxA1. Cell 122, 33-43 (2005).
43. Behbod, F. et al. An intraductal human-in-mouse transplantation model mimics the subtypes of ductal carcinoma in situ. Breast Cancer Res. 11, R66 (2009).

44. Sflomos, G. et al. A preclinical model for ERa-positive breast cancer points to the epithelial microenvironment as determinant of luminal phenotype and hormone response. Cancer Cell. 29, 407-422 (2016).

45. Kanchanawong, P. et al. Nanoscale architecture of integrin-based cell adhesions. Nature 468, 580-584 (2010).

46. Sage, D. et al. Quantitative evaluation of software packages for single-molecule localization microscopy. Nat. Methods 12, 717-724 (2015).

47. Chou, Y.-Y. et al. Identification and characterization of a novel broadspectrum virus entry inhibitor. J. Virol. 90, 4494-4510 (2016).

\section{Acknowledgements}

We wish to acknowledge the Experimental Mouse Resource Service, particularly Gillian Paine-Murrieta and Bethany Skovan for their technical assistance; Agnes Witkiewicz and the Tissue Acquisition and Cellular/Molecular Analysis (TACMSR), particularly Betsy Dennison for procuring patient samples; Paul Krieg (University of Arizona, Tucson, AZ, USA) for comments on the manuscript; the staff at the Advanced Imaging Center at Janelia Research Campus for their assistance with iPALM imaging, a facility generously supported by the Gordon and Betty Moore Foundation and the Howard Hughes Medical Institute; Jonathan Kaye (Cedars-Sinai, Los Angeles, CA, USA) for the Cedars-Sinai LumB TMA and the National Cancer Institute Cancer Diagnosis Program for the BCP TMA. This research was supported by the NCI grant RO1 CA196885-01 (to G.M.), diversity supplemental RO1 CA196885-01 grant (to J.I.P.), Science Foundation Arizona Bisgrove Scholars Postdoctoral Fellowship (to S.S.P.), and the NCI University of Arizona Cancer Center Support Grant P30CA023074.

\section{Author contributions}

G.M. and M.P.-R. designed the study and prepared the manuscript. M.P.-R., S.S.P., D.G.A., J.I.P., A.W.W., S.M.H., and J.A. performed and analyzed experiments. S.S.P., J.A., M.P.-R., and S.M.H. performed and analyzed the super-resolution imaging experiments at Janelia Research Campus. T.W. performed and analyzed the ChIP-seq and ChIP-PCR experiment. M.N. analyzed the TCGA data. R.G. generated the CRISPR cells. D.T. contributed to developing the LII. D.J.R. provided input on the statistical analyses. B.K provided reagents, and contributed intellectually to the analysis and interpretation of the data. M.P.-R., S.S.P., T.W., J.I.P., J.A., B.K., and G.M. wrote the manuscript.

\section{Additional information}

Supplementary Information accompanies this paper at https://doi.org/10.1038/s41467018-05367-2.

Competing interests: The authors declare no competing interests.

Reprints and permission information is available online at http://npg.nature.com/ reprintsandpermissions/

Publisher's note: Springer Nature remains neutral with regard to jurisdictional claims in published maps and institutional affiliations.

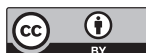

Open Access This article is licensed under a Creative Commons Attribution 4.0 International License, which permits use, sharing, adaptation, distribution and reproduction in any medium or format, as long as you give appropriate credit to the original author(s) and the source, provide a link to the Creative Commons license, and indicate if changes were made. The images or other third party material in this article are included in the article's Creative Commons license, unless indicated otherwise in a credit line to the material. If material is not included in the article's Creative Commons license and your intended use is not permitted by statutory regulation or exceeds the permitted use, you will need to obtain permission directly from the copyright holder. To view a copy of this license, visit http://creativecommons.org/ licenses/by/4.0/.

(C) The Author(s) 2018 